\title{
Focus on Undernutrition in Care Homes: A Retrospective Service Evaluation
}

\author{
Rachael Masters \\ Bishop Auckland Hospital, Cockton Hill Road, Bishop Auckland DL14, England
}

\begin{abstract}
Let them eat cake": a retrospective service evaluation of Focus on Undernutrition in care homes. Introduction: Undernutrition is a major cause and consequence of poor health in older people, affecting $35 \%$ of residents in care homes. Focus on Undernutrition (FoU), a dietetic service delivered by dietetic assistants uses a multifaceted approach to undernutrition management. This study aims to evaluate FoU's impact on undernutrition outcome measures in care homes; including FoU's influence on weight change in residents "at risk" of undernutrition, and prevalence of undernutrition and pressure ulcers (PU). Methods: A retrospective pragmatic service evaluation was undertaken using pseudonymised data collected over 13 years on weight, undernutrition risk and PU from long-stay residents' notes before and six months after training (FoU). Results: Analysis completed on 104 homes, 4,315 residents (71.3\% female; mean stay 10.8 (1-278) months) in County Durham. Following FoU a significant difference was identified for: improved rate of weight change for "at risk" residents $(p<0.001)$. Undernutrition risk significantly influenced weight change (low: ${ }^{\mathrm{B}} 1.04 \mathrm{~kg},{ }^{\mathrm{E}} 0.01 \mathrm{~kg}$; moderate: ${ }^{\mathrm{B}}-1.79 \mathrm{~kg},{ }^{\mathrm{E}}-0.38 \mathrm{~kg}$; high: ${ }^{\mathrm{B}}-0.83 \mathrm{~kg},{ }^{\mathrm{E}} 1.00 \mathrm{~kg},{ }^{\mathrm{B}} p<0.001,{ }^{\mathrm{E}} p=0.001$ ). Reduced undernutrition prevalence $(p<0.001)$ from $32.7 \%$ to $29.1 \%$ residents "at risk" of undernutrition (moderate: ${ }^{\mathrm{B}} 13.1 \%,{ }^{\mathrm{E}} 8.9 \%$; high: ${ }^{\mathrm{B}} 19.6 \%$, $\left.{ }^{\mathrm{E}} 15.9 \%\right)$. Nutrition screening significantly improved $\left({ }^{\mathrm{B}} 76.3 \%,{ }^{\mathrm{E}} 98.7 \%, p<0.001\right)$, reduced prevalence PU $(51 \%, p<0.001)$. PU prevalence significantly increased with undernutrition severity at baseline $(p<0.001)$, but not following FoU $(p=0.233)\left(\right.$ low: ${ }^{\mathrm{B}} 5 \%$, ${ }^{\mathrm{E}} 2.3 \%$; moderate: ${ }^{\mathrm{B}} 6.9 \%,{ }^{\mathrm{E}} 1.6 \%$; high: ${ }^{\mathrm{B}} 10.5 \%,{ }^{\mathrm{E}} 3.9 \%$ ). Odds of developing PU reduced $53 \%$ (OR: 0.47$) .{ }^{\mathrm{B}}$ :baseline; ${ }^{\mathrm{E}}$ :evaluation; OR: odds ratio. Conclusion: These results demonstrate dietetic assistants delivering FoU significantly improves weight, undernutrition and PU prevalence in care homes. Indicating FoU is an effective model for improving undernutrition outcomes, with the potential of reducing possible harm, such as PU in care homes.
\end{abstract}

Key words: "MUST"/“malnutrition universal screening tool”, care homes older people, undernutrition, malnutrition, training, food first, nutrition screening tool.

\section{Abbreviations and Glossary}

\begin{tabular}{|c|c|}
\hline $\begin{array}{l}\text { Nutritional } \\
\text { screening }\end{array}$ & $\begin{array}{l}\text { Nutritional screening is a rapid simple way of } \\
\text { identifying individuals at risk of undernutrition }\end{array}$ \\
\hline NST & Nutritional screening tool \\
\hline “MUST” & $\begin{array}{l}\text { "Malnutrition Universal Screening Tool". The } \\
\text { UK's preferred NST }\end{array}$ \\
\hline $\begin{array}{l}\text { Undernutrition } \\
\text { risk }\end{array}$ & $\begin{array}{l}\text { The category identified for a resident's risk of } \\
\text { undernutrition, this is either low, moderate or } \\
\text { high }\end{array}$ \\
\hline "At risk" & $\begin{array}{l}\text { Term used to refer to residents who are } \\
\text { identified as either moderate or high risk of } \\
\text { undernutrition }\end{array}$ \\
\hline FoU & Focus on undernutrition \\
\hline $\mathrm{U}$ & Pressure ulcer \\
\hline & $\begin{array}{l}\text { Food based interventions. The use of } \\
\text { nourishing snacks and drinks, fortified meal } \\
\text { dishes and homemade fortified drinks used to } \\
\text { increase a resident's energy and nutritional } \\
\text { intake }\end{array}$ \\
\hline
\end{tabular}

Corresponding author: Rachael Masters, master, specialist dietitian, research field: undernutrition.
$\mathrm{kg}$
NSW
B
E

\begin{abstract}
Kilograms
Nutritional screening week. A national survey over 4 years conducted by BAPEN (British Association of Parenteral and Enteral Nutrition) to collect data on undernutrition risk in adults on admission to hospitals, mental health trusts and care homes across the UK. Baseline results. This period incorporates relevant data from admission to the date of baseline data collection.

Evaluation results. This period incorporates relevant data between the dates of baseline to evaluation data collections.
\end{abstract}

\section{Introduction}

Undernutrition is a silent epidemic [1], identified as a significant public health problem [2] and health inequality concern [3-5]. In the UK undernutrition affects 3 million adults [6], 1.3 million older people [6] and $35 \%$ of residents in care homes [7]. It is a major 
cause and consequence of poor health in older people [8-10], detrimentally affecting the functions of all bodily organs with serious consequences [2, 3], causing increased morbidity [11-14], reduced quality of life [13-17] and increased mortality [11-13, 18].

Annual costs associated with undernutrition in England are $£ 19.6$ billion [2]. The cost of caring for an undernourished individual is 2-4 times that of a well-nourished individual $[19,20]$ due to the strain on health and social care resources [2, 3]. Most people do not realize how common undernutrition is or the seriousness of its consequences [9], so it continues to be under-recognised and under-treated [3, 7-9, 19, 21, 22 ], to the detriment and cost of individuals and the health economy [3].

Undernutrition is largely preventable and treatable
[23-25] through pro-active screening and early intervention, which can reduce complications by $70 \%$ and mortality by $40 \%$ [13], thereby reducing or abolishing many of its detrimental clinical and functional consequences [2]. NICE [26] identified that pro-active management of undernutrition has the third highest potential to deliver cost savings from all NICE guidelines $[8,26]$. Prevention is key to achieving this $[24,27]$ through integrating screening and treatment into routine practices [27-29].

Over recent years over 20 standards, guidelines, toolkits and campaigns on undernutrition have been launched to raise the awareness in frontline staff, service providers and policy makers [30]. These all recommend similar best practice principles as outlined in Table 1.

Table 1 Overview of the best practice principles for the management of undernutrition from national guidance.

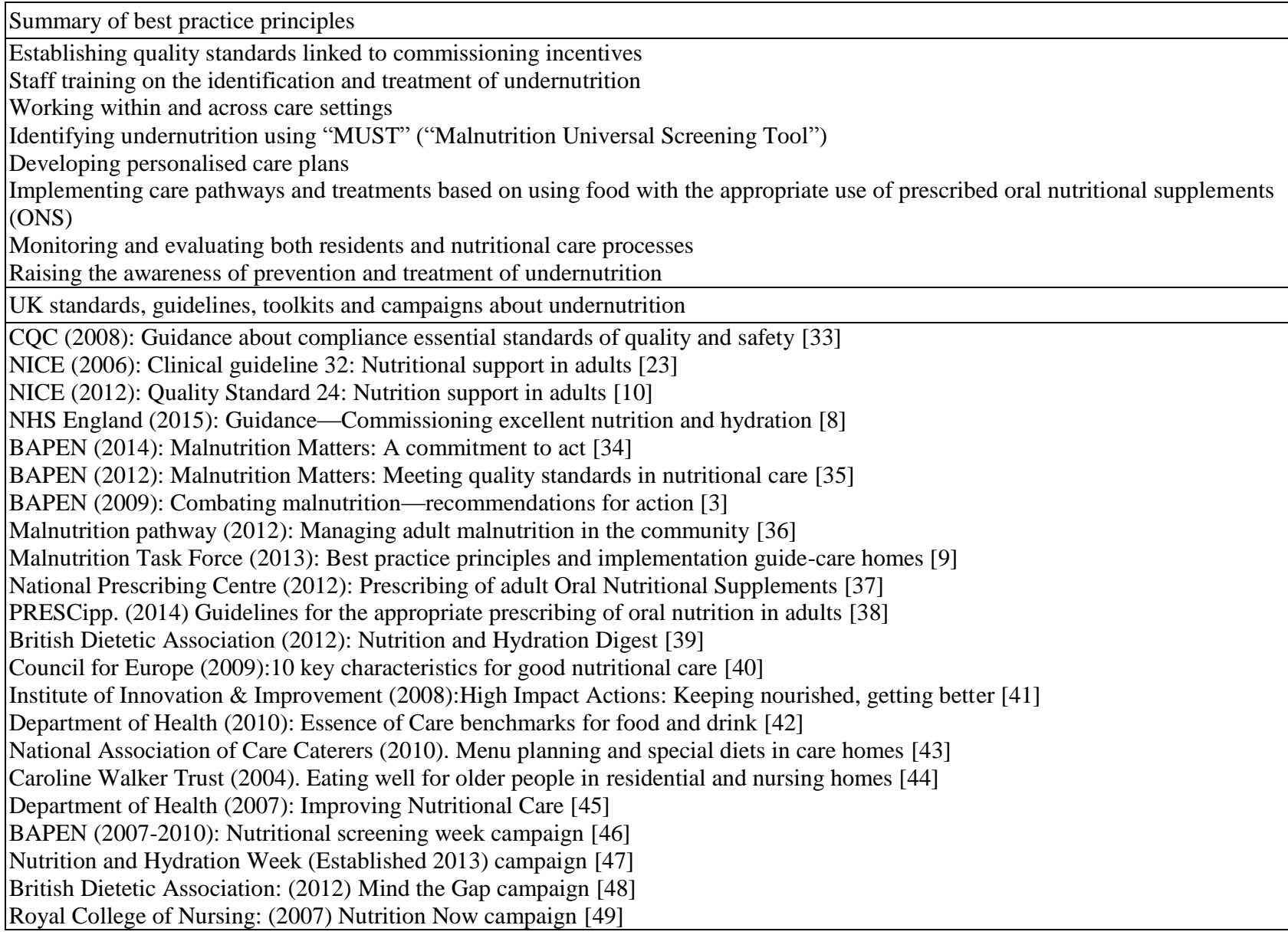

Yet despite all this guidance there is still a discrepancy between policy and practice [3, 31]. 
Undernutrition is under-detected and under-treated [3, $7,8]$; one in six care homes fail to meet minimum nutritional standards [32]; with the quality of undernutrition care being insufficient in care homes [27]. Reliable and sustainable improvements in the provision of nutritional care are needed to help reduce avoidable harm and improve patient outcomes and experience [30].

In 2000 prior to these national initiatives (Table 1), a pilot called "Focus on Food" (later renamed Focus on Undernutrition (FoU)) was implemented into care homes in North East England to determine the most effective way to facilitate the implementation of nutritional screening and guidelines into care homes [50]. The pilot was one of the first in England to implement practices in care homes which have now become accepted practice across the UK $[9,36-39,51$, 52], these included the implementation of:

- "Malnutrition Universal Screening Tool" "MUST" [53] to identify residents at risk of undernutrition. FoU simplified and adapted the "MUST" layout; which is endorsed by BAPEN; incorporating a results table, core care plan, weight and ulna height conversion charts and a dietary assessment [54].

- Standardised care pathways for the treatment of undernutrition, which promoted food based interventions $(\mathrm{FB})$ as treatment rather than oral nutritional supplements (ONS) [55].

- Standardised care pathways for appropriate ONS prescribing, prior to a dietetic referral [56].

- Dietitian role as an educator facilitator, delivering proactive training to empower care home staff to identify and treat undernutrition.

- Quality standards (Appendix 1) for undernutrition which were incorporated into the Local Authority Commission incentives linked to funding.

The results of the pilot identified open learning workbooks, supported by workshops, as the most effective method of implementing "MUST", with nutritional care declining significantly in homes which received no facilitation [50]. This pilot established the ethos for the current FoU delivery model.

In 2003 following the pilot, FoU was established as a permanent part of the dietetic service in County Durham and Darlington. FoU provides free training to care homes on the identification and treatment of undernutrition. Following training, homes implement FoU's adapted "MUST" [54]; identified by independent research to be more effective than original "MUST" [57] and care pathways [55, 56] which fulfill national guidance $[9,36-38]$ promoting FB interventions alongside the appropriate prescribing of ONS. Further information on FoU can be found online [58].

Despite FoU being nationally acknowledged as an exemplary service model $[8,37,59,60]$ no formal evidence exists of FoU's impact on undernutrition in care homes regardless of collecting evaluation data, due to problems with FoU's database since 2008.

The aim of this project was to evaluate the FoU service to determine the impact on undernutrition outcome measures in care homes.

The objectives were to determine if FoU influences the:

- weight of residents at risk of undernutrition

- prevalence of undernutrition

- prevalence of pressure ulcers.

\section{Methodology}

A retrospective service evaluation of 104 care homes in County Durham and Darlington which received FoU between 2003 and December 2015. Data were collected and inputted into FoU's database for 4,416 residents, of which 4,315 are included in the service evaluation. Inclusion and exclusion criteria are outlined in Table 2.

County Durham and Darlington NHS Foundation Trust (CDDFT) advised no formal submission or ethical approval was required due to the project being 
Table 2 Inclusion and exclusion criteria.

\begin{tabular}{|c|c|c|}
\hline & Inclusion & Exclusion \\
\hline Data collection & $\begin{array}{l}\text { A registered care home for older people within } \\
\text { County Durham or Darlington. }\end{array}$ & $\begin{array}{l}\text { Registered care homes for the young disabled or those with } \\
\text { learning difficulties. } \\
\text { A care home outside County Durham or Darlington. } \\
\text { A care home which fails to provide written consent. } \\
\text { Short term residents (such as respite, intermediate care) due to } \\
\text { being discharged prior to evaluation. }\end{array}$ \\
\hline Data analysis & $\begin{array}{l}\text { The completed resident data collection form } \\
\text { contained information on some key parameters } \\
\text { (such as weight, nutritional screening, pressure } \\
\text { ulcers) at baseline and/or evaluation. }\end{array}$ & $\begin{array}{l}\text { The completed resident data collection form did not contain } \\
\text { information on any key parameters (such as weight, nutritional } \\
\text { screening, pressure ulcers) at baseline and/or evaluation. }\end{array}$ \\
\hline
\end{tabular}

Table 3 Parameters collected and analysed from resident records.

\begin{tabular}{|c|c|c|c|}
\hline & & Before training & $\begin{array}{l}6 \text { months } \\
\text { after training }\end{array}$ \\
\hline \multirow{5}{*}{ Demographic } & Age range at baseline & & $\sqrt{ }$ \\
\hline & Gender & & $\sqrt{ }$ \\
\hline & Care status (residential, nursing, EMI) & & $\sqrt{ }$ \\
\hline & Presence of dementia & & $\sqrt{ }$ \\
\hline & Date of admission & & $\sqrt{ }$ \\
\hline \multirow{2}{*}{$\begin{array}{l}\text { Anthropometric } \\
\text { information }\end{array}$} & Weight $(\mathrm{kg})$ on admission & $\sqrt{ }$ & \\
\hline & Weights $(\mathrm{kg})$ for the previous 6 months & $\sqrt{ }$ & $\sqrt{ }$ \\
\hline \multirow{3}{*}{$\begin{array}{l}\text { Nutritional screening } \\
\text { information }\end{array}$} & Risk of undernutrition using care home nutritional screening tool & $\sqrt{ }$ & \\
\hline & Risk of undernutrition using FoU “MUST" completed by DA & $\sqrt{ }$ & \\
\hline & Risk of undernutrition (FoU “MUST”) for previous 6 months & & $\sqrt{ }$ \\
\hline \multirow[b]{2}{*}{ Other information } & Is the resident prescribed oral nutritional supplements (ONS)? & $\sqrt{ }$ & $\sqrt{ }$ \\
\hline & Is the resident referred to the dietitian? & $\sqrt{ }$ & $\sqrt{ }$ \\
\hline
\end{tabular}

a retrospective service evaluation. Written consent was provided by the home managers on behalf of the homes to collect established data from resident records for the purpose of service evaluation. An information leaflet and copy of consent were given to the manager.

\subsection{Data Collection}

The stages of FoU delivery are outlined in Appendix 2, these are implemented in accordance with standard operating procedures. A competency trained dietetic assistant collected pseudonymised data from all long-stay resident records before and six months after training, using standardised data collection sheets (Appendix 3). No data were collected which was not part of routine records. Table 3 outlines the data analyzed in this service evaluation, all other data collected (Appendix 3) are out of scope.

\subsection{Data Analysis}

Fig. 1 outlines the stages of data preparation and analysis; significant challenges were experienced with merging databases and data cleansing. The final dataset for analysis was created by combining two source datasets; a "legacy dataset" which held data from 2003 to 2008 and the "current dataset" which held data from 2009 onwards. Prior to merging, the datasets were cleansed and verified. Data syntax was normalized between the datasets for each field and then the datasets were de-duped and merged. The resulting dataset was prepared which employed syntax that the Statistical Package for Social Sciences, version 22 [SPSS Chicago, USA] could analyze. No missing data could be recollected because of the time 
1. Update FoU Access database to fulfill current and future service requirements

- Role: External consultant and principle investigator

- Date: Jan 2015-April 2015

2. Input retrospective data onto new FoU database (data from 2008-end 2015)

- Role: Dietetic Assistant

- Date: May 2015-Feb 2016

\section{Merging "legacy" and "current" FoU databases using Excel}

- Role: External consultant and principle investigator

- Date: March 2016

\section{Data cleansing merged database using Excel}

- Role: External consultant and principle investigator

- Date: March-April 2016

5. Analysis and coding data using Excel suitable for importing to SPSS

- Role: External consultant and principle investigator

- Date: May 2016

\section{Statistical analysis using SPSS}

- Role: Principle investigator with advice from statisticians at Newcastle University

- Date: June-July 2016

- Continuous weight data: one way and split plot analysis of the variance (ANOVA), paired

and independent $t$-tests. All data was checked for normality (Kolmogorov Smirnov, $p<0.05$ ) and homogeneity of variance (Leven's, $p=>0.05$ ). Kruskall-Wallis test used when data normally distributed but Leven's, $p<0.05$.

- Categorical data for the prevalence of undernutrition and pressure ulcers: Chi-square

- Inter-observer agreement between the care home and FoU nutritional screening results:

Cohen's Kappa

- Effect of FoU training on the presence of pressure ulcers according to nutritional risk : Odds ratio

Fig. 1 Overview of the stages for data inputting, cleansing and analysis.

delay between data collection and inputting. Data collection, storage and analysis fulfilled CDDFT's information risk management policy.

Statistical advice was sought from Newcastle University. Weight was calculated as rate of weight change, represented as kilogram per month, due to the confounding impact of duration. The majority of data were analyzed as independent samples, because paired data were only available for $49 \%$ of residents. Fig. 1 , stage 6 identifies the statistical tests used to analyze data. Continuous data are represented as mean, standard deviation, minimum-maximum and 95\% confidence intervals. Categorical data are reported as number and percentage frequencies. All reported $p$ 
values are based on two-sided tests and compared to a significance level of $5 \%(p<0.05)$. Significant refers to statistical significance, as identified by the correct statistical analysis reporting a result of $p<0.05$.

\subsection{Study Population}

Retrospective data were analyzed from 2003-December 2015, incorporating 104 care homes and 4,315 residents. Figs. 2 and 3 outline that data were

\section{Results}

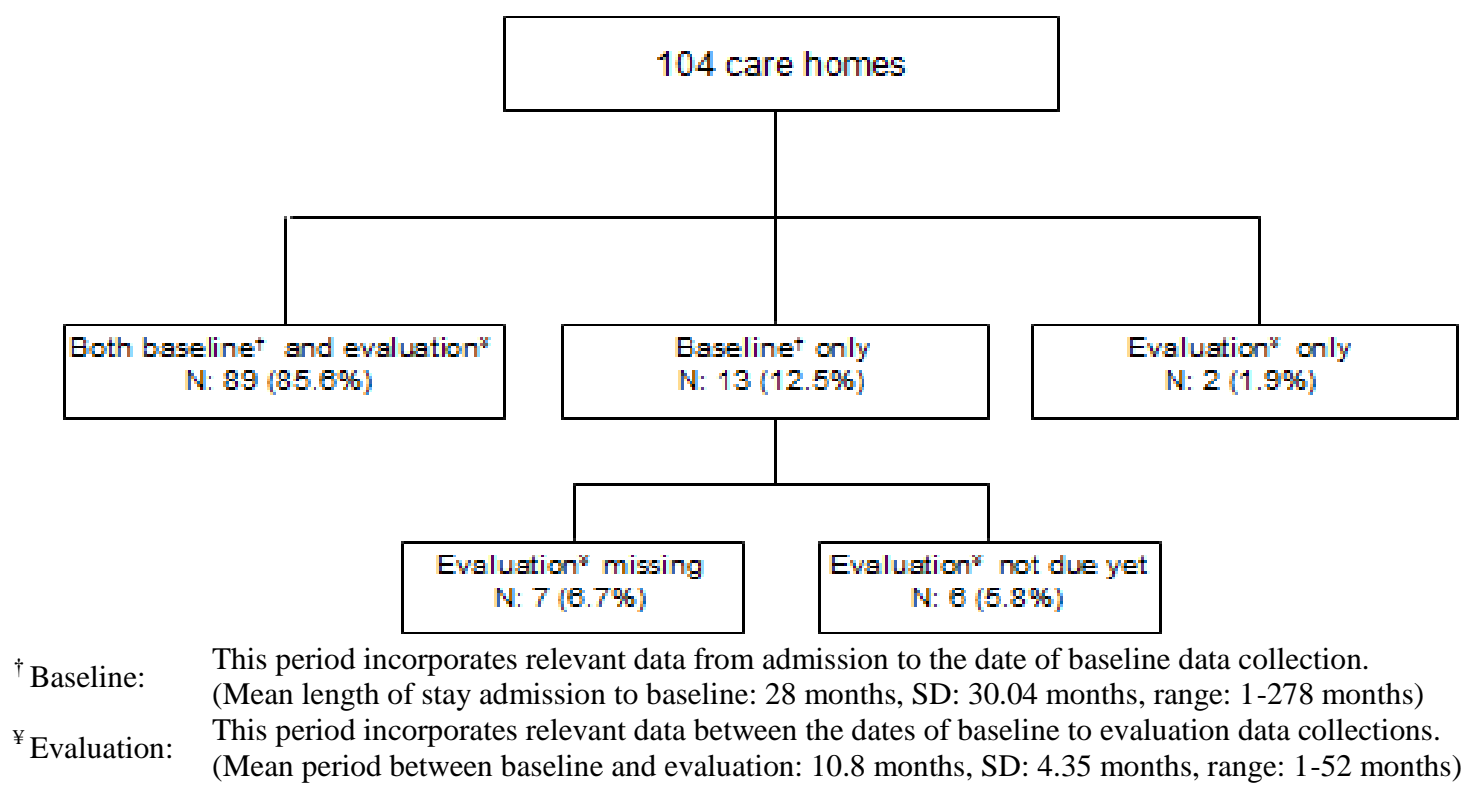

Fig. 2 Data inclusion for care homes.

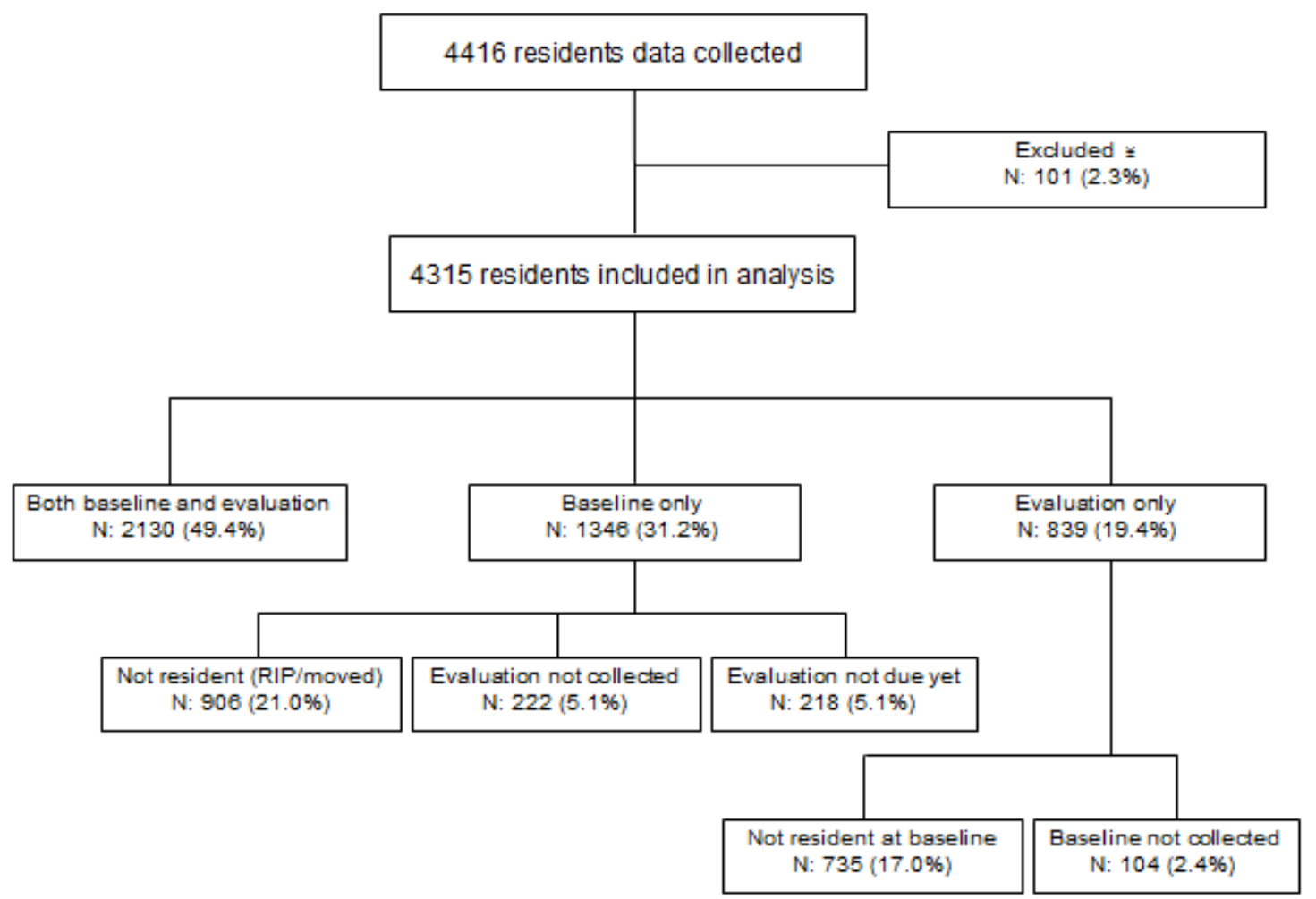

Fig. 3 Data inclusion for residents.

$¥$ Excluded from analysis because poor data quality on key variables (e.g. weight, “MUST”, pressure ulcers). 
available for $85.6 \%$ of homes and $49.4 \%$ of residents at both baseline and evaluation; $101(2.3 \%)$ residents were excluded because of poor quality data. Care home demographics are presented in Table 4, 38.5\% were dual registered, $5.8 \%$ of homes subsequently closed. Table 5 shows residents were predominately

Table 4 Care home demographics at baseline.

\begin{tabular}{llll}
\hline Demographic & Category & Number & Percentage \\
\hline \multirow{2}{*}{ Registered care category } & Nursing and residential & 40 & $38.5 \%$ \\
& Nursing and/or residential and EMI* & 35 & $33.7 \%$ \\
& Residential & 24 & $23.0 \%$ \\
Open or & Nursing & 5 & $4.8 \%$ \\
closed & Open & 98 & $94.2 \%$ \\
Phase of & Closed & $6.8 \%$ \\
FoU delivery & $2003-2008$ (Individual delivery) & 19 & $18.3 \%$ \\
\hline
\end{tabular}

* EMI: Elderly Mentally Ill Care Home.

Table 5 Resident demographics at baseline.

\begin{tabular}{|c|c|c|c|c|c|c|c|}
\hline Demographic & Category & Numbe & & & Percentage & & \\
\hline \multirow[t]{3}{*}{ Gender } & Female & 3,075 & & & $71.3 \%$ & & \\
\hline & Male & 1,240 & & & $28.7 \%$ & & \\
\hline & & $\mathrm{T}$ & $\mathrm{F}$ & M & $\mathrm{T}$ & $\mathrm{F}$ & M \\
\hline \multirow{7}{*}{ Age range at baseline } & $<65$ & 78 & 31 & 47 & $2.2 \%$ & $1.2 \%$ & $4.6 \%$ \\
\hline & $65-69$ & 102 & 45 & 57 & $2.8 \%$ & $1.8 \%$ & $5.6 \%$ \\
\hline & $70-79$ & 610 & 370 & 240 & $17.0 \%$ & $14.4 \%$ & $23.4 \%$ \\
\hline & $80-89$ & 1,614 & 1153 & 461 & $44.9 \%$ & $45.0 \%$ & $44.9 \%$ \\
\hline & $90-99$ & 1,082 & 875 & 207 & $30.1 \%$ & $34.1 \%$ & $20.2 \%$ \\
\hline & $>100$ & 105 & 91 & 14 & $2.9 \%$ & $3.5 \%$ & $1.4 \%$ \\
\hline & Unknown ${ }^{*}$ & 724 & & & - & & \\
\hline \multirow[t]{4}{*}{ Care category } & Residential & 2,291 & & & $55.3 \%$ & & \\
\hline & Nursing & 1,035 & & & $25.0 \%$ & & \\
\hline & EMI* & 817 & & & $19.7 \%$ & & \\
\hline & Unknown ${ }^{*}$ & 172 & & & - & & \\
\hline \multirow[t]{2}{*}{ Dementia } & Yes & 1,645 & & & $38.1 \%$ & & \\
\hline & No & 2,670 & & & $61.9 \%$ & & \\
\hline \multirow{7}{*}{$\begin{array}{l}\text { Average length stay to } \\
\text { baseline }\end{array}$} & $<1$ year & 1,135 & & & $38.1 \%$ & & \\
\hline & $1-2$ years & 606 & & & $20.3 \%$ & & \\
\hline & $2-3$ years & 440 & & & $14.8 \%$ & & \\
\hline & 3-4 years & 322 & & & $10.8 \%$ & & \\
\hline & $4-5$ years & 144 & & & $4.8 \%$ & & \\
\hline & $>5$ years & 333 & & & $11.2 \%$ & & \\
\hline & Unknown ${ }^{*}$ & 1,335 & & & - & & \\
\hline \multirow{2}{*}{ Mean length of stay } & 28 months (SD: $30.04 \mathrm{~m}$ ) & & & & & & \\
\hline & Min-Max: 1-278 months & & & & & & \\
\hline Phase of & $\begin{array}{l}\text { 2003-2008 (Individual } \\
\text { delivery) }\end{array}$ & 704 & & & $16.3 \%$ & & \\
\hline FoU delivery & 2009-2015 (Team delivery) & 3611 & & & $83.7 \%$ & & \\
\hline
\end{tabular}

*EMI: Elderly Mentally Ill Care Category. SD: Standard deviation. T: Total; F: Female; M: Male.

${ }^{\ddagger}$ Unknown: Percentage (number) of unknown data for the variable; age: $16.8 \%$ (724); care category: $4 \%$ (172); length of stay: $30.9 \%$ $(1,335)$. Unknown data was excluded from the analysis, all percentages are representative of known data. 
female (71.3\%), 92\% aged between 70-100 years; residential being the most frequent care type (55.3\%). Although " $<1$ year" category was the most frequent duration $(38.1 \%)$, mean stay was 28 months.

\subsection{Weight Change}

Following FoU the overall average rate of weight change increased (B: $0.007 \mathrm{~kg} /$ month, E: 0.002 $\mathrm{kg} / \mathrm{month}$ ), but this was not significant (Table 6). Neither dementia, care type (Table 6), or gender $\left(\mathrm{B}^{5}: p\right.$ $\left.=0.539, \mathrm{E}^{5}: p=0.265\right)$ had a significant impact on weight change. Duration was only significant following FoU (B: $p^{2}=0.969$, E: $p^{2}=0.021$ ).

Following FoU a significant improvement in weight change was shown for "at risk" residents (Table 6, Fig. 4). A significant difference was identified between undernutrition risk at baseline $(p<0.001)$, evaluation $(p=0.009)$ and overall $(p<0.001)$, which was significant both within residents $(p<0.001)$ and between groups $(p<0.001)$ (Table 6$)$. The greatest weight change was reflected in high risk residents (absolute change: $0.29 \mathrm{~kg} / \mathrm{month}$ ).

To compare findings alongside literature, actual and percentage weight change were analysed. Undernutrition risk was significantly associated with actual weight change (Table 7), similar to the findings on rate of weight change (Fig. 4, Table 6).

A reversal in percentage weight change was identified in "at risk" residents, most noticeable in high risk improving from $-4.4 \%$ to $+2.4 \%$. There was a significant difference between baseline and evaluation, and according to risk group (Table 8).

Weight loss of >10\% over 6 months is a determinant for undernutrition [23]. Undernutrition risk was significantly associated $(p<0.001)$ with percentage weight change; $66.9 \%$ of high risk residents lost weight compared to $42.3 \%$ of low risk, with the greatest weight improvements seen in high risk residents (Table 9).

Further analysis of "at risk" residents according to treatment identified all treatment options reversed from weight loss to weight gain after FoU, with the greatest improvements seen in residents prescribed ONS, but no significant difference $(p=0.399)$ was found between treatments (Table 6, Fig. 5). Only 28.3\% (208/735) and 31.1\% (127/409) of "at risk" residents were prescribed ONS at baseline and evaluation respectively. Of residents prescribed ONS, 32.2\% $(101 / 314)$ were prescribed them both at baseline and evaluation.

\subsection{Undernutrition Prevalence}

Overall there was a significant reduction (11.0\%) in the undernutrition prevalence following FoU (Fig. 6, Table 10).

Undernutrition was significantly more prominent in female residents ( $p<0.001$ ), which may be associated with greater age (Table 5); a significant difference was shown in undernutrition prevalence according to age category (B: $p=0.005$, E: $p=0.001$ ). A significant difference was found in undernutrition prevalence according to care category ( $p<0.001$ ), highest prevalence being identified in nursing $(40.6 \%)$, but all categories demonstrated a reduction in undernutrition following FoU (Table 10). Although residents with dementia had an increased prevalence of undernutrition, this was only significant at evaluation $(p=0.001)$ despite prevalence declining in both groups (Table 10). Duration only significantly influenced prevalence at baseline $\left(\mathrm{B}: p^{1}=0.013, \mathrm{E}: p^{1}\right.$ $=0.326$ ).

Further analysis of "at risk" residents according to treatment identified the greatest improvements in the "no treatment" group (45.7\%). Overall 14.2\% (45/316) of "at risk" residents experienced a decline, $42.4 \%$ (134/316) improvement and 43.4\% (137/316) no change in undernutrition risk (Fig. 7).

A significant improvement $(p<0.001)$ in undertaking nutritional screening was identified following FoU, increasing from $76.3 \%$ to $98.7 \%$ (Fig. 8). However, there is only fair agreement [62] $(\mathrm{k}=$ 0.33 ) between the care home and FoU for identifying 
Table 6 Rate of weight change (kg/month).

\begin{tabular}{|c|c|c|c|c|c|c|c|c|c|c|c|}
\hline & & & \multirow{2}{*}{$\begin{array}{l}\text { Mean } \\
\text { (kg/month) }\end{array}$} & \multirow{2}{*}{ SD } & \multirow{2}{*}{ Min } & \multirow{2}{*}{ Max } & \multicolumn{2}{|c|}{$95 \% \mathrm{CI}$} & \multirow[t]{2}{*}{$\mathrm{N}$} & \multirow[t]{2}{*}{$\%$} & \multirow[t]{2}{*}{ Statistics } \\
\hline & & & & & & & Lower & Upper & & & \\
\hline \multirow{2}{*}{\multicolumn{2}{|c|}{$\begin{array}{l}\text { Average rate of weight } \\
\text { change (kg/month) }\end{array}$}} & B & -0.007 & 0.75 & -13.3 & 13.9 & - & - & 2627 & $71 \%$ & \multirow{2}{*}{$\begin{array}{l}\mathrm{B}-\mathrm{E}^{1}: \\
p=0.583\end{array}$} \\
\hline & & $\mathrm{E}$ & 0.002 & 0.42 & -3.5 & 6.4 & - & - & 2,627 & $87.8 \%$ & \\
\hline \multirow{6}{*}{ "MUST"* } & \multirow[t]{2}{*}{ Low } & B & 0.074 & 0.79 & -6.8 & 13.9 & 0.03 & 0.12 & 1,437 & $67.8 \%$ & \multirow{6}{*}{$\begin{array}{l}\mathrm{B}^{2}: p<0.001 \\
\mathrm{E}^{2}: p=0.009 \\
\mathrm{~B}-\mathrm{E}^{3}: p<0.001 \\
\text { Within subjects } \\
p<0.001 \\
\text { Between groups } \\
p<0.001\end{array}$} \\
\hline & & $\mathrm{E}$ & -0.006 & 0.45 & -3.3 & 3.9 & -0.03 & 0.02 & 1,437 & $67.8 \%$ & \\
\hline & \multirow[t]{2}{*}{ Moderate } & B & -0.124 & 0.86 & -11.6 & 1.6 & -0.22 & -0.03 & 278 & $13.1 \%$ & \\
\hline & & $\mathrm{E}$ & -0.018 & 0.46 & -.35 & 1.7 & -0.07 & 0.04 & 278 & $13.1 \%$ & \\
\hline & \multirow[t]{2}{*}{ High } & B & -0.204 & 0.89 & -13.8 & 3.9 & -0.28 & -0.13 & 406 & $19.1 \%$ & \\
\hline & & $\mathrm{E}$ & 0.052 & 0.47 & -1.3 & 6.4 & 0.01 & 0.10 & 406 & $19.1 \%$ & \\
\hline \multirow{6}{*}{ Care category } & \multirow[t]{2}{*}{ Residential } & B & -0.001 & 0.74 & -13.8 & 5.3 & -0.038 & 0.04 & 1,505 & $56.9 \%$ & \multirow{6}{*}{$\begin{array}{l}\mathrm{B}^{2}: p=0.400 \\
\mathrm{E}^{2}: p=0.496\end{array}$} \\
\hline & & $\mathrm{E}$ & 0.012 & 0.42 & -3.5 & 6.4 & -0.01 & 0.03 & 1,991 & $54.5 \%$ & \\
\hline & Nursing & B & -0.022 & 0.73 & -3.0 & 7.6 & -0.08 & 0.04 & 615 & $23.2 \%$ & \\
\hline & \multirow{3}{*}{ EMI } & $\mathrm{E}$ & -0.003 & 0.36 & -2.5 & 2.0 & -0.03 & 0.02 & 901 & $24.7 \%$ & \\
\hline & & B & -0.049 & 0.70 & -5.4 & 8.5 & -0.11 & 0.01 & 527 & $19.9 \%$ & \\
\hline & & E & -0.003 & 0.39 & -2.6 & 3.7 & -0.03 & 0.03 & 759 & $20.8 \%$ & \\
\hline \multirow{4}{*}{ Dementia } & Yes & B & -0.003 & 0.67 & -11.6 & 7.6 & -0.036 & 0.030 & 1,596 & $58.0 \%$ & \multirow{4}{*}{$\begin{array}{l}\mathrm{B}^{5}: p=0.448 \\
\mathrm{E}^{5}: p=0.451\end{array}$} \\
\hline & \multirow{3}{*}{ No } & $\mathrm{E}$ & 0.009 & 0.40 & -3.5 & 5.6 & -0.007 & 0.025 & 2,323 & $61.3 \%$ & \\
\hline & & B & -0.020 & 0.80 & -13.8 & 8.5 & -0.066 & 0.026 & 1,157 & $42.0 \%$ & \\
\hline & & $\mathrm{E}$ & -0.001 & 0.39 & -2.6 & 6.4 & -0.021 & 0.019 & 1,466 & $38.7 \%$ & \\
\hline \multirow{6}{*}{$\begin{array}{l}\text { Treatment } \\
\text { option for "at } \\
\text { risk" * of } \\
\text { undernutrition }\end{array}$} & \multirow{2}{*}{$\begin{array}{l}\text { (1) No } \\
\text { treatment }\end{array}$} & B & -0.094 & 0.64 & -5.4 & 5.3 & -0.16 & -0.03 & 381 & $69.4 \%$ & \multirow{6}{*}{$\begin{array}{l}B^{6}: p=0.770 \\
E^{2}: p=0.399\end{array}$} \\
\hline & & $\mathrm{E}$ & 0.037 & 0.50 & -2.6 & 5.6 & -0.02 & 0.09 & 323 & $68.7 \%$ & \\
\hline & (2) Only & B & -0.315 & 1.58 & -11.6 & 1.3 & -0.73 & 0.10 & 57 & $10.4 \%$ & \\
\hline & ONS & $\mathrm{E}$ & 0.058 & 0.29 & -0.8 & 1.1 & -0.02 & 0.14 & 51 & $10.9 \%$ & \\
\hline & \multirow{2}{*}{$\begin{array}{l}\text { (3) ONS \& } \\
\text { dietitian }\end{array}$} & B & -0.168 & 0.73 & -3.6 & 3.9 & -0.31 & -0.03 & 111 & $20.2 \%$ & \\
\hline & & E & 0.122 & 0.72 & -1.0 & 6.4 & -0.02 & 0.27 & 96 & $20.4 \%$ & \\
\hline \multirow{3}{*}{$\begin{array}{l}\text { Change in } \\
\text { nutritional status }\end{array}$} & Improved & - & 0.212 & 0.74 & -3.5 & 6.4 & 0.12 & 0.30 & 260 & $14.0 \%$ & \multirow{3}{*}{$\begin{array}{l}{ }^{2}: p<0.001 \\
{ }^{4}: p<0.001\end{array}$} \\
\hline & Stable & - & 0.050 & 0.48 & -3.3 & 3.9 & 0.02 & 0.08 & 1,286 & $69.3 \%$ & \\
\hline & Declined & - & -0.309 & 0.45 & -2.2 & 1.9 & -0.36 & -0.26 & 309 & $16.7 \%$ & \\
\hline
\end{tabular}

B: Baseline, the period between the date of admission and date of baseline data collection.

Mean length of stay: 28 months (m), SD: 30.4m, range: 1-278 m.

E: Evaluation, the period between baseline and evaluation data collections.

Mean period: 10.8 months (m), SD: $4.3 \mathrm{~m}$, range: $1-52 \mathrm{~m}$.

EMI: Elderly Mentally Ill care category.

SD: Standard deviation.

ONS: Oral Nutritional Supplements, a prescribed nutritional drink used to treat undernutrition.

* "MUST": Malnutrition Universal Screening Tool, used to identify risk of undernutrition. "At risk" of undernutrition is a term based on a combination of moderate and high risk residents.

${ }^{1}$ : Paired $t$-test. Normality (Kolmogorov-Smirnov) and homogeneity of the variance (Leven's) test completed.

2: One way ANOVA. Normality (Kolmogorov-Smirnov) and homogeneity of the variance (Leven's) test completed.

3. Split plot ANOVA multivariate. Normality (Kolmogorov-Smirnov) \& homogeneity of variance (Leven's) test completed.

4: Split plot ANOVA identified significant difference between "within residents" and "between risk groups".

5: Unpaired t-test. Normality (Kolmogorov-Smirnov) and homogeneity of the variance (Leven's) test completed.

${ }^{6}$ : Kruskall-Wallis test used for baseline because homogeneity of the variance was significant (Leven's $p=0.020$ ). 


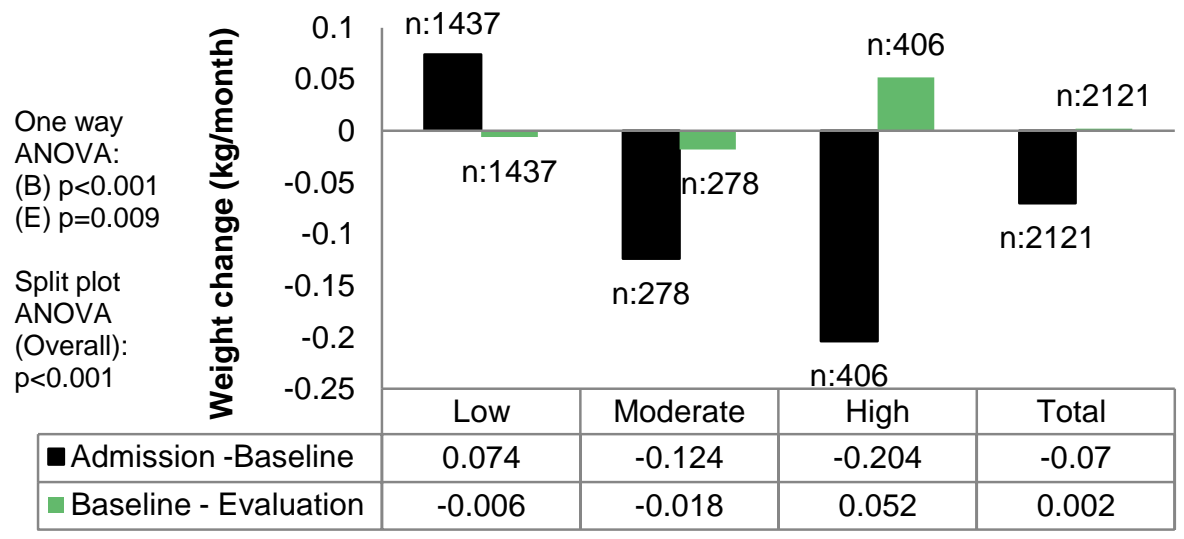

Fig. 4 Rate of weight change according to undernutrition risk.

Table 7 Overall actual weight change (kg) according to undernutrition risk.

\begin{tabular}{|c|c|c|c|c|c|c|c|c|c|c|c|}
\hline & & & \multirow{2}{*}{$\begin{array}{l}\text { Mean } \\
(\mathrm{kg})\end{array}$} & \multirow{2}{*}{$\begin{array}{l}\text { SD } \\
(\mathrm{kg})\end{array}$} & \multirow{2}{*}{$\begin{array}{l}\text { Min } \\
(\mathrm{kg})\end{array}$} & \multirow{2}{*}{$\begin{array}{l}\text { Max } \\
(\mathrm{kg})\end{array}$} & \multicolumn{2}{|c|}{$95 \% \mathrm{CI}(\mathrm{kg})$} & \multirow{2}{*}{$\mathrm{N}$} & \multirow{2}{*}{$\%$} & \multirow{2}{*}{ Statistics } \\
\hline & & & & & & & Lower & Upper & & & \\
\hline \multirow{2}{*}{\multicolumn{2}{|c|}{ Actual weight change $(\mathrm{kg})$}} & B & 0.55 & 7.10 & -59.7 & 61.0 & - & - & 1561 & $36.2 \%$ & \multirow{2}{*}{$\mathrm{B}-\mathrm{E}^{1}: p=0.813$} \\
\hline & & $\mathrm{E}$ & 0.11 & 5.16 & -46.1 & 50.0 & - & - & 1561 & $36.2 \%$ & \\
\hline \multirow{6}{*}{ "MUST" * } & Low & B & 1.04 & 6.72 & -24.2 & 32.5 & 0.64 & 1.44 & 1076 & $72.1 \%$ & \multirow{6}{*}{$\begin{array}{l}\mathrm{B}^{2}: p<0.001 \\
\mathrm{E}^{2}: p<0.001 \\
\mathrm{~B}-\mathrm{E}^{3}: \\
p<0.001 \\
\text { Within subjects }^{4} \\
p<0.001 \\
\text { Between groups } \\
p<0.001\end{array}$} \\
\hline & & $\mathrm{E}$ & 0.01 & 4.96 & -26.1 & 35.4 & -0.29 & 0.30 & 1076 & $72.1 \%$ & \\
\hline & Moderate & B & -1.79 & 7.20 & -41.4 & 24.2 & -2.85 & -0.74 & 181 & $12.1 \%$ & \\
\hline & & $\mathrm{E}$ & -0.38 & 5.82 & -46.1 & 15.1 & -1.23 & 0.47 & 181 & $12.1 \%$ & \\
\hline & \multirow[t]{2}{*}{ High } & B & -2.83 & 6.66 & -59.7 & 15.4 & -3.68 & -1.97 & 236 & $15.8 \%$ & \\
\hline & & $\mathrm{E}$ & 1.00 & 5.01 & -7.7 & 44.6 & 0.36 & 1.64 & 236 & $15.8 \%$ & \\
\hline
\end{tabular}

B: Baseline, the period between the date of admission and date of baseline data collection.

Mean length of stay: 28 months (m), SD: $30.4 \mathrm{~m}$, range: 1-278 m.

E: Evaluation, the period between baseline and evaluation data collections.

Mean period: 10.8 months (m), SD: $4.3 \mathrm{~m}$, range: 1-52 m.

SD: Standard deviation.

* "MUST": Malnutrition Universal Screening Tool, used to identify risk of undernutrition. "At risk" of undernutrition is a term based on a combination of moderate and high risk residents.

1: Paired $t$-test. Normality (Kolmogorov-Smirnov) and homogeneity of the variance (Leven's) test completed.

2 : One way ANOVA. Normality (Kolmogorov-Smirnov) and homogeneity of the variance (Leven's) test completed.

3: Split plot ANOVA multivariate. Normality (Kolmogorov-Smirnov) and homogeneity of the variance (Leven's) test completed.

4: Split plot ANOVA identified significant difference both between "within residents" and "between risk groups".

undernutrition risk. There was $60 \%$ agreement overall, with the least agreement at moderate risk (29.2\%) and best at low risk (68.8\%) (Table 11). Comparing agreement was only completed at baseline.

\subsection{Pressure Ulcers}

Following FoU pressure ulcers (PU) overall significantly reduced 51\% $(p<0.001)$ (Table 12). At baseline there was a significant difference in PU prevalence which increased with severity of undernutrition ( $p<0.001$ ), but following FoU no difference was found between risk groups $(p=0.233)$ (Fig. 9).

Undernutrition risk appears to have an impact on PU; the odds overall of developing a PU following FoU was $53 \%$ less, with the true population effect between $64 \%$ and $38 \%$. The greatest reduction $(78 \%)$ was in moderate risk residents. The odds identified a significant reduction in $\mathrm{PU}$ in all risk groups (Table 13). 
Table 8 Average percentage weight change according to undernutrition risk.

\begin{tabular}{|c|c|c|c|c|c|c|c|c|c|c|c|}
\hline & & & \multirow{2}{*}{$\begin{array}{l}\text { Mean } \\
(\%)\end{array}$} & \multirow{2}{*}{$\begin{array}{l}\text { SD } \\
(\%)\end{array}$} & \multirow{2}{*}{$\begin{array}{l}\text { Min } \\
(\%)\end{array}$} & \multirow{2}{*}{$\begin{array}{l}\operatorname{Max} \\
(\%)\end{array}$} & \multicolumn{2}{|c|}{$95 \%$ CI (\%) } & \multirow{2}{*}{$\mathrm{N}$} & \multirow{2}{*}{$\%$} & \multirow{2}{*}{ Statistics } \\
\hline & & & & & & & lower & upper & & & \\
\hline \multirow{2}{*}{\multicolumn{2}{|c|}{$\begin{array}{l}\text { Percentage weight change } \\
(\%)\end{array}$}} & B & $0.67 \%$ & 11.55 & -60.2 & 132.5 & - & - & 1,561 & $36.2 \%$ & \multirow{2}{*}{$\begin{array}{l}\mathrm{B}-\mathrm{E}^{1}: \\
p=0.583\end{array}$} \\
\hline & & E & $0.47 \%$ & 8.60 & -50.5 & 139.0 & - & - & 1,561 & $36.2 \%$ & \\
\hline \multirow{6}{*}{ "MUST" * } & \multirow[t]{2}{*}{ Low } & B & $2.30 \%$ & 11.02 & -30.9 & 132.5 & 1.64 & 2.96 & 1,076 & $72.1 \%$ & \multirow{6}{*}{$\begin{array}{l}\mathrm{B}^{2}: p<0.001 \\
\mathrm{E}^{2}: p<0.001 \\
\mathrm{~B}-\mathrm{E}^{3}: \\
p<0.001 \\
\text { Within subjects } \\
p<0.001 \\
\text { Between groups } \\
p<0.001\end{array}$} \\
\hline & & E & $0.16 \%$ & 7.52 & -33.4 & 51.1 & -0.35 & 0.67 & 1,076 & $72.1 \%$ & \\
\hline & \multirow[t]{2}{*}{ Moderate } & B & $-2.18 \%$ & 11.26 & -43.1 & 50.1 & -3.80 & -0.57 & 181 & $12.1 \%$ & \\
\hline & & E & $-0.28 \%$ & 9.44 & -50.5 & 30.1 & -1.52 & 0.96 & 181 & $12.1 \%$ & \\
\hline & \multirow[t]{2}{*}{ High } & B & $-4.42 \%$ & 11.11 & -57.0 & 40.3 & -5.83 & -3.01 & 236 & $15.8 \%$ & \\
\hline & & E & $2.42 \%$ & 11.39 & -16.5 & 139.0 & 1.34 & 3.50 & 236 & $15.8 \%$ & \\
\hline
\end{tabular}

B: Baseline, the period between the date of admission and date of baseline data collection.

Mean length of stay: 28 months (m), SD: $30.4 \mathrm{~m}$, range: 1-278 m.

E: Evaluation, the period between baseline and evaluation data collections.

Mean period: 10.8 months (m), SD: $4.3 \mathrm{~m}$, range: $1-52 \mathrm{~m}$.

SD: Standard deviation.

* "MUST": Malnutrition Universal Screening Tool, used to identify risk of undernutrition. "At risk" of undernutrition is a term based on a combination of moderate and high risk residents.

${ }^{1}$ : Paired $t$-test. Normality (Kolmogorov-Smirnov) and homogeneity of the variance (Leven's) test completed.

2: One way ANOVA. Normality (Kolmogorov-Smirnov) and homogeneity of the variance (Leven's) test completed.

${ }^{3}$ : Split plot ANOVA multivariate. Normality (Kolmogorov-Smirnov) and homogeneity of the variance (Leven's) test completed.

4: Split plot ANOVA identified significant difference both between "within residents" and "between risk groups".

Table 9 Weight change categorised as $>10 \%, 5-10 \%$ according to undernutrition risk.

\begin{tabular}{|c|c|c|c|c|c|c|c|c|}
\hline & & & \multicolumn{2}{|c|}{ Weight gain } & \multicolumn{3}{|c|}{ Weight loss } & \multirow[t]{2}{*}{ Statistics } \\
\hline & & & $\begin{array}{l}>10 \% \\
\%(\mathrm{n})\end{array}$ & $\begin{array}{l}5-10 \% \\
\%(\mathrm{n})\end{array}$ & $\begin{array}{l}>10 \% \\
\%(\mathrm{n})\end{array}$ & $\begin{array}{l}5-10 \% \\
\%(\mathrm{n})\end{array}$ & $\begin{array}{l}\text { Overall } \\
\text { weight loss }\end{array}$ & \\
\hline \multirow[t]{2}{*}{ Overall } & & B & $\begin{array}{l}13.1 \% \\
(305)\end{array}$ & $\begin{array}{l}9.7 \% \\
(226)\end{array}$ & $\begin{array}{l}14.6 \% \\
(340)\end{array}$ & $\begin{array}{l}12.3 \% \\
(285)\end{array}$ & $\begin{array}{l}49.3 \% \\
(1,146)\end{array}$ & \multirow[t]{2}{*}{$\mathrm{B}-\mathrm{E}^{1}: p<0.001$} \\
\hline & & $\mathrm{E}$ & $\begin{array}{l}9.3 \% \\
(171)\end{array}$ & $\begin{array}{l}12.0 \% \\
(220)\end{array}$ & $\begin{array}{l}7.7 \% \\
(141)\end{array}$ & $\begin{array}{l}13.0 \% \\
(238)\end{array}$ & $\begin{array}{l}48.8 \% \\
(895)\end{array}$ & \\
\hline \multirow[t]{7}{*}{ "MUST"* } & \multirow[t]{2}{*}{ Low } & B & $\begin{array}{l}15.6 \% \\
(244)\end{array}$ & $\begin{array}{l}11.3 \% \\
(117)\end{array}$ & $\begin{array}{l}9.4 \% \\
(147)\end{array}$ & $\begin{array}{l}10.1 \% \\
(158)\end{array}$ & $\begin{array}{l}42.3 \% \\
(662)\end{array}$ & \multirow[t]{7}{*}{$\begin{array}{l}\mathrm{B}^{1}: p<0.001 \\
\mathrm{E}^{1}: P<0.001\end{array}$} \\
\hline & & $\mathrm{E}$ & $\begin{array}{l}7.6 \% \\
(100)\end{array}$ & $\begin{array}{l}10.9 \% \\
(143)\end{array}$ & $\begin{array}{l}7.3 \% \\
(96)\end{array}$ & $\begin{array}{l}12.8 \% \\
(168)\end{array}$ & $\begin{array}{l}49.9 \% \\
(654)\end{array}$ & \\
\hline & \multirow[t]{2}{*}{ Moderate } & B & $\begin{array}{l}10.8 \% \\
(33)\end{array}$ & $\begin{array}{l}6.2 \% \\
(19)\end{array}$ & $\begin{array}{l}19.7 \% \\
(60)\end{array}$ & $\begin{array}{l}17.0 \% \\
(52)\end{array}$ & $\begin{array}{l}60.3 \% \\
(184)\end{array}$ & \\
\hline & & $\mathrm{E}$ & $\begin{array}{l}8.1 \% \\
(18)\end{array}$ & $\begin{array}{l}15.2 \% \\
(34)\end{array}$ & $\begin{array}{l}9.4 \% \\
(21)\end{array}$ & $\begin{array}{l}17.0 \% \\
(38)\end{array}$ & $\begin{array}{l}52.4 \% \\
(117)\end{array}$ & \\
\hline & \multirow{3}{*}{ High } & $B$ & $6.3 \%$ & $6.7 \%$ & $29.7 \%$ & $16.7 \%$ & $66.9 \%$ & \\
\hline & & D & $(28)$ & $(30)$ & (133) & $(75)$ & $(300)$ & \\
\hline & & $\mathrm{E}$ & $\begin{array}{l}17.4 \% \\
(53)\end{array}$ & $\begin{array}{l}14.1 \% \\
(43)\end{array}$ & $\begin{array}{l}7.9 \% \\
(24)\end{array}$ & $\begin{array}{l}10.5 \% \\
(32)\end{array}$ & $\begin{array}{l}40.7 \% \\
(124)\end{array}$ & \\
\hline
\end{tabular}

$\%$ : Percentage of residents; (n) Number of residents.

1: Chi-Square Test.

* "MUST": Malnutrition Universal Screening Tool, used to identify risk of undernutrition. "At risk" of undernutrition is a term based on a combination of moderate and high risk residents.

$\mathrm{B}$ : Baseline, the period between the date of admission and date of baseline data collection.

Mean length of stay: 28 months (m), SD: $30.4 \mathrm{~m}$, range: 1-278 m.

E: Evaluation, the period between baseline and evaluation data collections.

Mean period: 10.8 months (m), SD: $4.3 \mathrm{~m}$, range: $1-52 \mathrm{~m}$. 


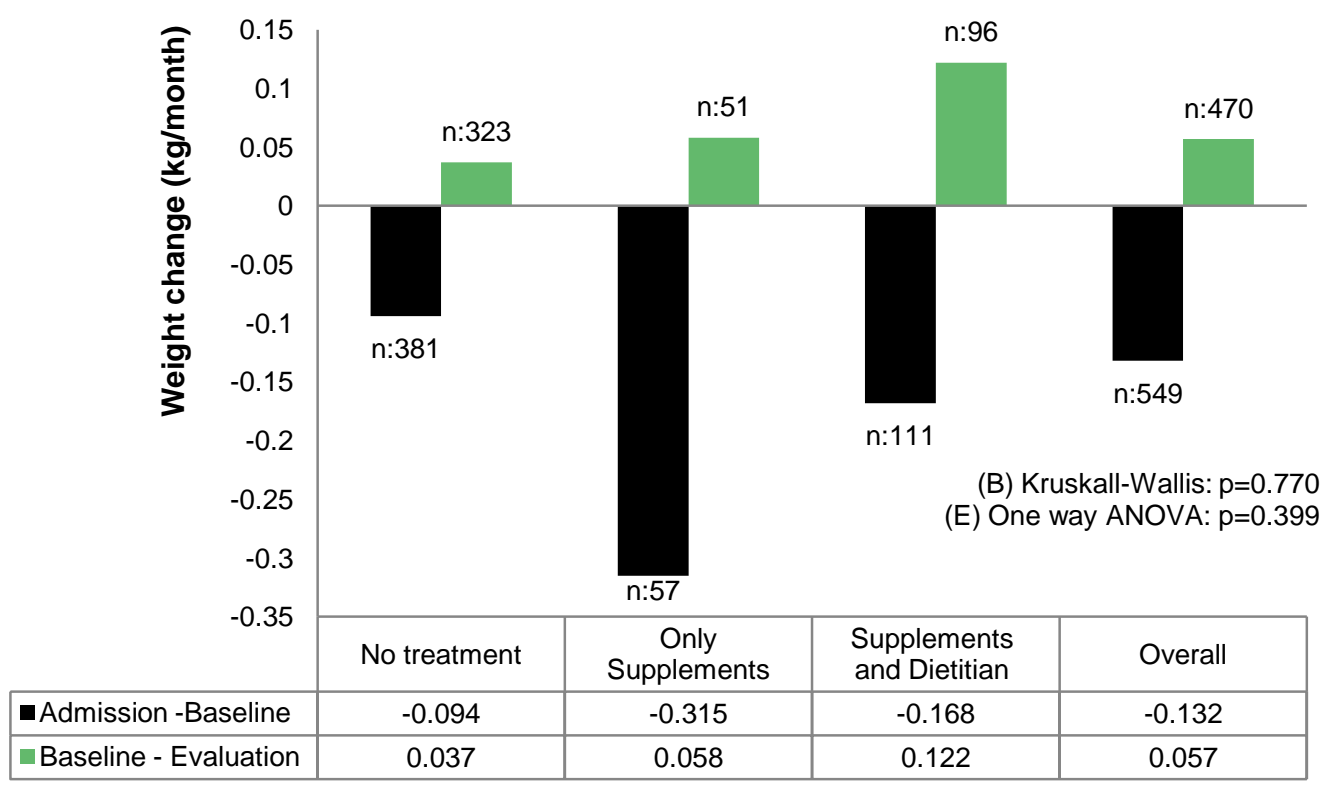

Fig. 5 Rate of weight change in "at risk" residents according to treatment.

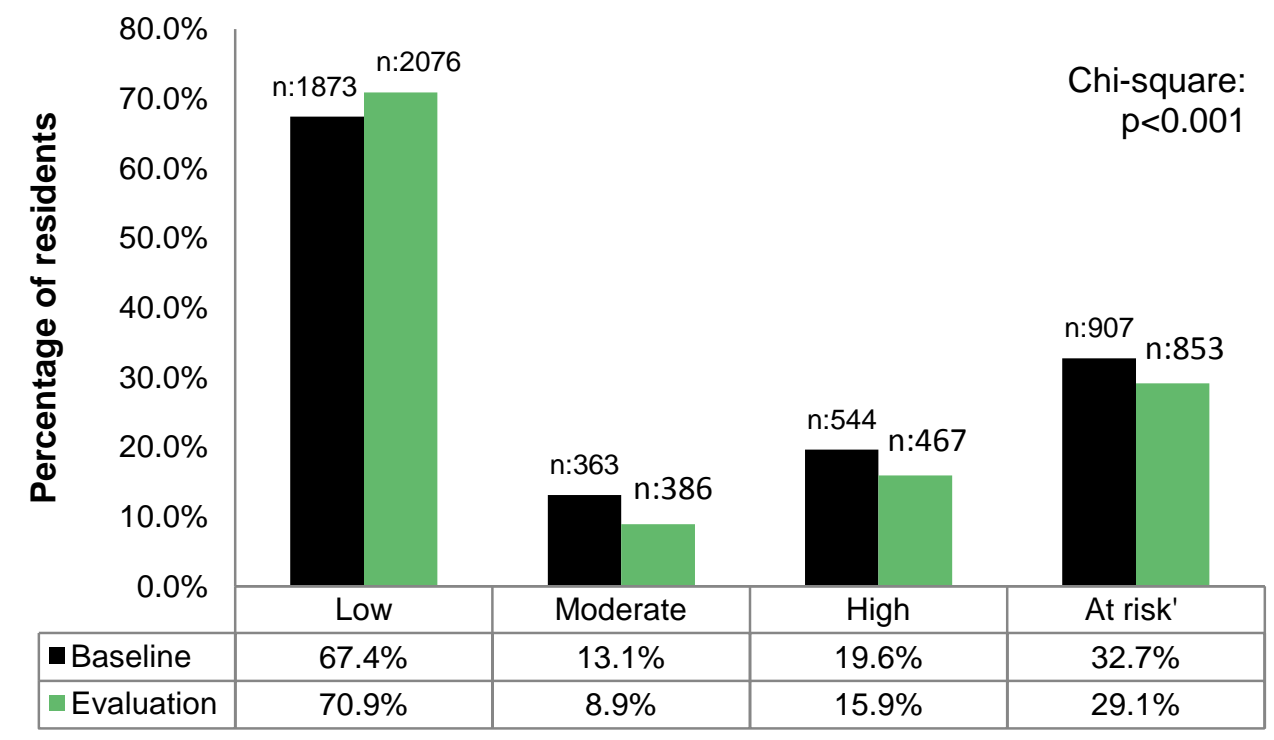

Fig. 6 Prevalence of undernutrition. 
Table 10 Prevalence of undernutrition according to different variables.

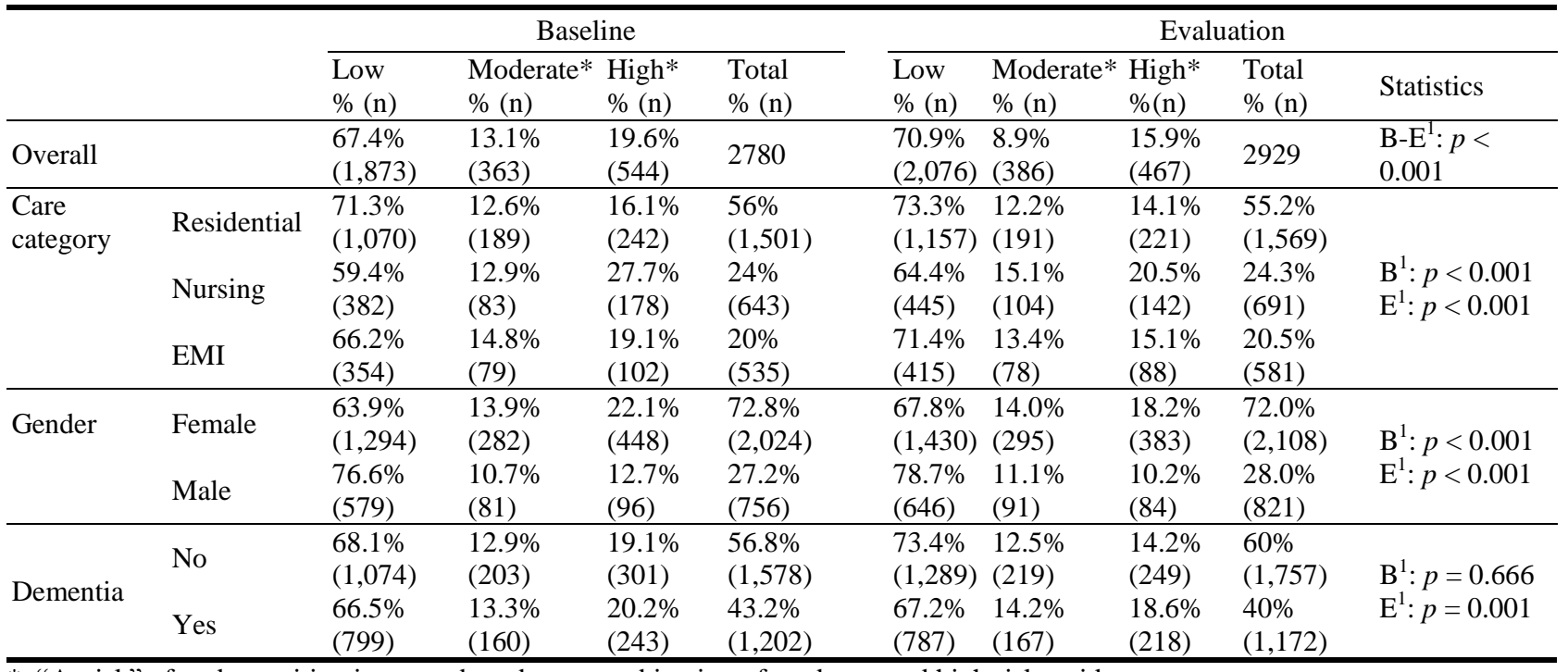

*: "At risk" of undernutrition is a term based on a combination of moderate and high risk residents.

$\%$ : Percentage of residents; (n) Number of residents; ${ }^{1}$ : Chi-Square Test.

$\mathrm{B}$ : Baseline, the period between the date of admission and date of baseline data collection.

Mean length of stay: 28 months (m), SD: $30.4 \mathrm{~m}$, range: 1-278 m.

E: Evaluation, the period between baseline and evaluation data collections.

Mean period: 10.8 months (m), SD: $4.3 \mathrm{~m}$, range: $1-52 \mathrm{~m}$.

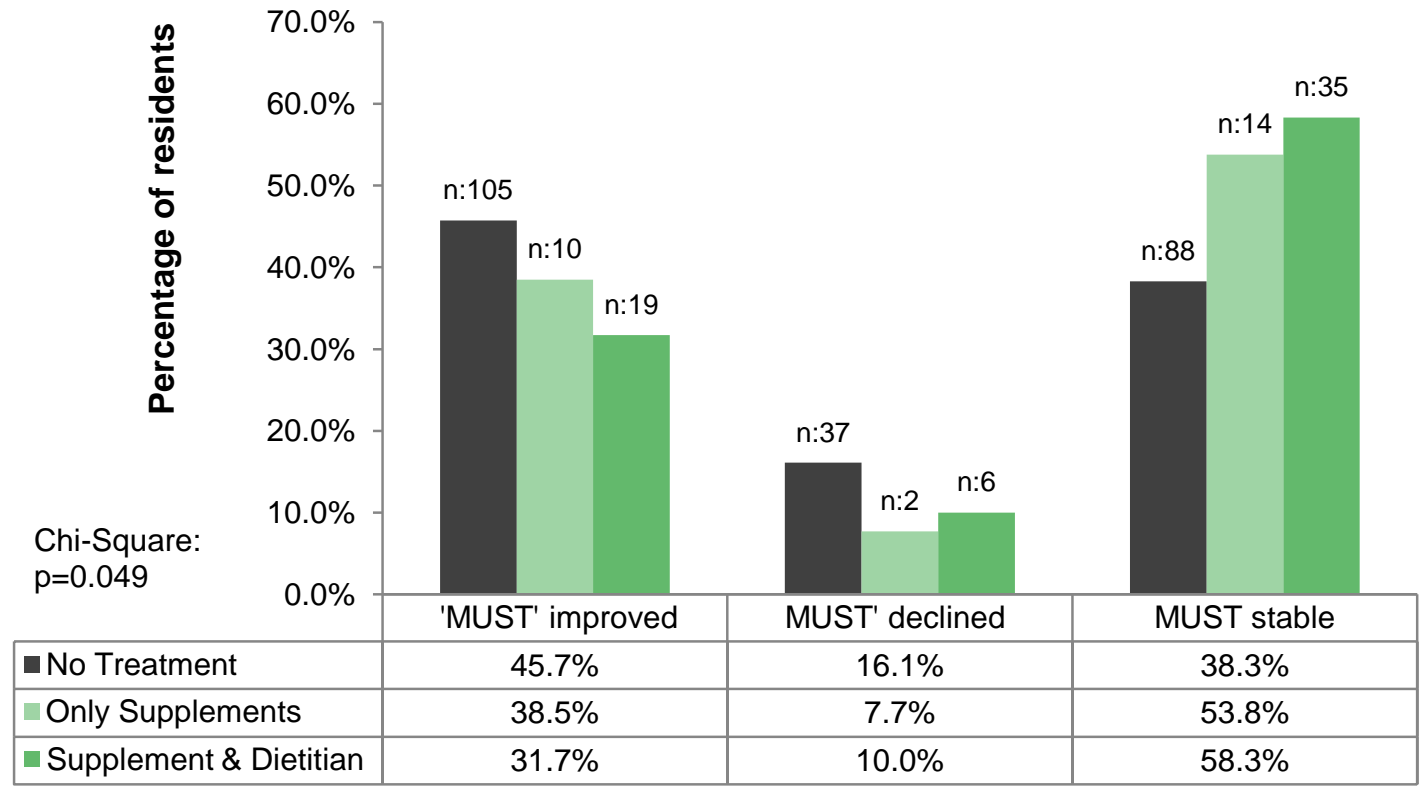

Fig. 7 Changes in undernutrition risk in "at risk" residents according to treatment. 


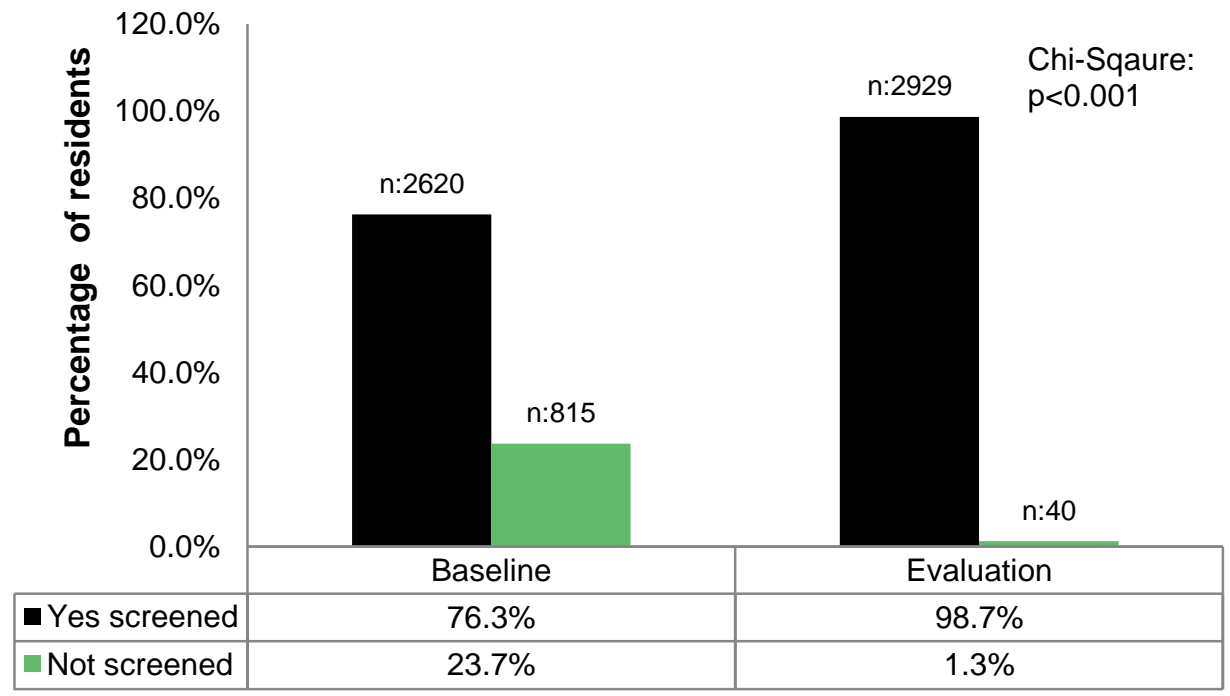

Fig. 8 Completion of nutritional screening.

Table 11 Inter-observer agreement between care home and FoU identifying undernutrition risk.

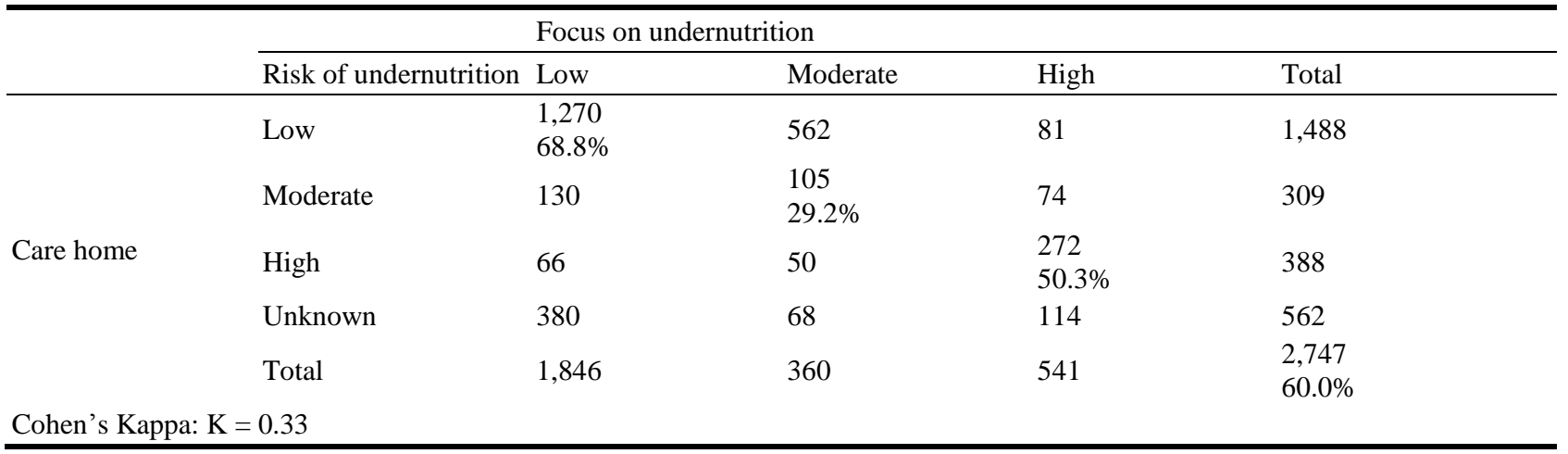

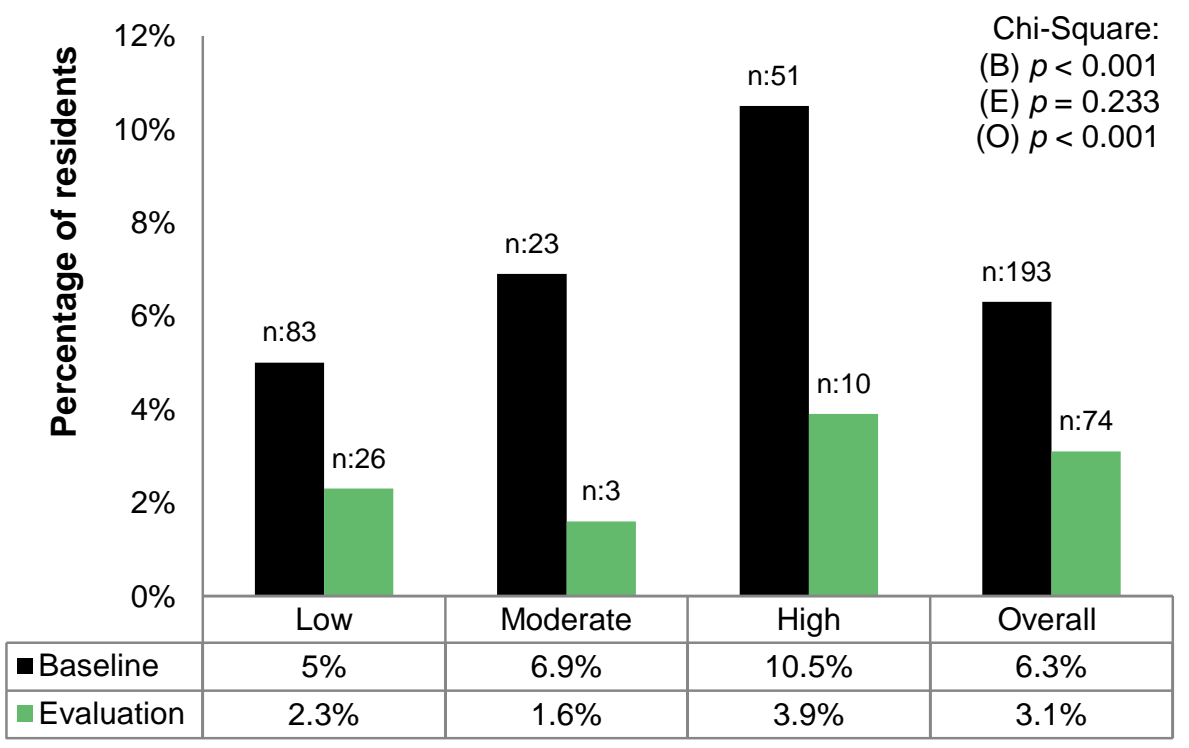

Fig. 9 Prevalence of pressure ulcers according to undernutrition risk. 
Table 12 Prevalence of pressure ulcers according to different variables.

\begin{tabular}{|c|c|c|c|c|c|c|}
\hline & & & ressure area & & pressure a & \\
\hline & & $\begin{array}{l}\text { Yes } \\
\%(\mathrm{n})\end{array}$ & $\begin{array}{l}\text { No } \\
\%(\mathrm{n})\end{array}$ & $\begin{array}{l}\text { Yes } \\
\%(\mathrm{n})\end{array}$ & $\begin{array}{l}\text { No } \\
\%(n)\end{array}$ & Statistics \\
\hline Overall & & $\begin{array}{l}6.3 \% \\
(193)\end{array}$ & $\begin{array}{l}93.7 \% \\
(2,880)\end{array}$ & $\begin{array}{l}3.1 \% \\
(74)\end{array}$ & $\begin{array}{l}96.9 \% \\
(2,352)\end{array}$ & ${ }^{1}: p<0.001$ \\
\hline & Low & $\begin{array}{l}5 \% \\
(83)\end{array}$ & $\begin{array}{l}95 \% \\
(1,576)\end{array}$ & $\begin{array}{l}2.3 \% \\
(26)\end{array}$ & $\begin{array}{l}97.7 \% \\
(1,088)\end{array}$ & \\
\hline Risk of undernutrition & Moderate & $\begin{array}{l}6.9 \% \\
(23)\end{array}$ & $\begin{array}{l}93.1 \% \\
(310)\end{array}$ & $\begin{array}{l}1.6 \% \\
\text { (3) }\end{array}$ & $\begin{array}{l}98.4 \% \\
(188)\end{array}$ & $\begin{array}{l}\mathrm{B}^{1}: p<0.001 \\
\mathrm{E}^{1}: p=0.233\end{array}$ \\
\hline & High & $\begin{array}{l}10.5 \% \\
(51)\end{array}$ & $\begin{array}{l}89.5 \% \\
(433)\end{array}$ & $\begin{array}{l}3.9 \% \\
(10)\end{array}$ & $\begin{array}{l}96.1 \% \\
(245) \\
\end{array}$ & \\
\hline Care Category & Residential & $\begin{array}{l}5.1 \% \\
(85)\end{array}$ & $\begin{array}{l}94.9 \% \\
(1,581)\end{array}$ & $\begin{array}{l}2.3 \% \\
(29)\end{array}$ & $\begin{array}{l}97.7 \% \\
(1,254)\end{array}$ & \\
\hline & Nursing & $\begin{array}{l}10.0 \% \\
(77)\end{array}$ & $\begin{array}{l}90.0 \% \\
(695)\end{array}$ & $\begin{array}{l}5.6 \% \\
(33)\end{array}$ & $\begin{array}{l}94.4 \% \\
(560)\end{array}$ & $\begin{array}{l}\mathrm{B}^{1}: p<0.001 \\
\mathrm{E}^{1}: p<0.001\end{array}$ \\
\hline & EMI* & $\begin{array}{l}5.1 \% \\
(27) \\
\end{array}$ & $\begin{array}{l}94.9 \% \\
(500)\end{array}$ & $\begin{array}{l}2.2 \% \\
(10)\end{array}$ & $\begin{array}{l}97.8 \% \\
(453) \\
\end{array}$ & \\
\hline Treatment option for "at & No treatment & $\begin{array}{l}9.1 \% \\
(37)\end{array}$ & $\begin{array}{l}90.9 \% \\
(370)\end{array}$ & $\begin{array}{l}3.1 \% \\
(7)\end{array}$ & $\begin{array}{l}96.9 \% \\
(218)\end{array}$ & \\
\hline risk" undernutrition & Only ONS & $\begin{array}{l}8.2 \% \\
(5)\end{array}$ & $\begin{array}{l}91.8 \% \\
(56)\end{array}$ & $\begin{array}{l}0 \% \\
(0)\end{array}$ & $\begin{array}{l}100 \% \\
(26)\end{array}$ & $\begin{array}{l}\mathrm{B}^{1}: p=0.105 \\
\mathrm{E}^{1}: p=0.580\end{array}$ \\
\hline & ONS \& dietitian & $\begin{array}{l}15.7 \% \\
(18)\end{array}$ & $\begin{array}{l}84.3 \% \\
(97)\end{array}$ & $\begin{array}{l}1.8 \% \\
\text { (1) }\end{array}$ & $\begin{array}{l}98.2 \% \\
(56)\end{array}$ & \\
\hline Dementia & Yes & $\begin{array}{l}6.7 \% \\
(93)\end{array}$ & $\begin{array}{l}93.3 \% \\
(1,296)\end{array}$ & $\begin{array}{l}3.0 \% \\
(34)\end{array}$ & $\begin{array}{l}97.0 \% \\
(1,117)\end{array}$ & $\mathrm{B}^{1}: p=0.762$ \\
\hline & No & $\begin{array}{l}5.9 \% \\
(100)\end{array}$ & $\begin{array}{l}94.1 \% \\
(1,584)\end{array}$ & $\begin{array}{l}3.1 \% \\
(40)\end{array}$ & $\begin{array}{l}96.9 \% \\
(1,235)\end{array}$ & $\mathrm{E}^{1}: p=0.793$ \\
\hline
\end{tabular}

$\%$ : Percentage of residents; (n) Number of residents. ': Chi-Square Test.

B: Baseline (Period between the date of admission and date of baseline data collection).

E: Evaluation (Period between baseline and evaluation data collections).

ONS: Oral Nutritional Supplements, a prescribed nutritional drink used to treat undernutrition.

Table 13 Odds ratio for pressure damage according to nutritional risk.

\begin{tabular}{llllll}
\hline \multirow{2}{*}{ Risk of undernutrition* } & \multirow{2}{*}{ Odds Ratio } & \multirow{2}{*}{$\begin{array}{l}\text { Reduction in pressure } \\
\text { ulcers after training }\end{array}$} & \multicolumn{3}{c}{ 95\% CI OR } \\
\cline { 4 - 6 } Low & 0.45 & $55 \%$ & 0.29 & Upper & $p=0.001$ \\
Moderate & 0.22 & $78 \%$ & 0.06 & 0.73 & $p=0.001$ \\
High & 0.35 & $65 \%$ & 0.17 & 0.69 & $p=0.003$ \\
Overall & 0.47 & $53 \%$ & 0.36 & 0.62 & $p<0.001$ \\
\hline
\end{tabular}

* Risk of undernutrition based on "MUST". CI: Confidence Interval. OR: Odds Ratio.

Although following FoU there was a reduction in PU for all care categories, a significant difference $(p<$ 0.001 ) in prevalence was seen in nursing compared to other catergories. There was no difference in prevalence in residents with dementia $(p=0.762, p=$ 0.793) (Table 12).
Further analysis on PU prevalence in "at risk residents" according to treatment identified those receiving ONS with dietetic care had the greatest reduction, but sample size was limited and no significance was found between treatments $(p=0.105$, $p=0.580$ ) (Fig. 10). 


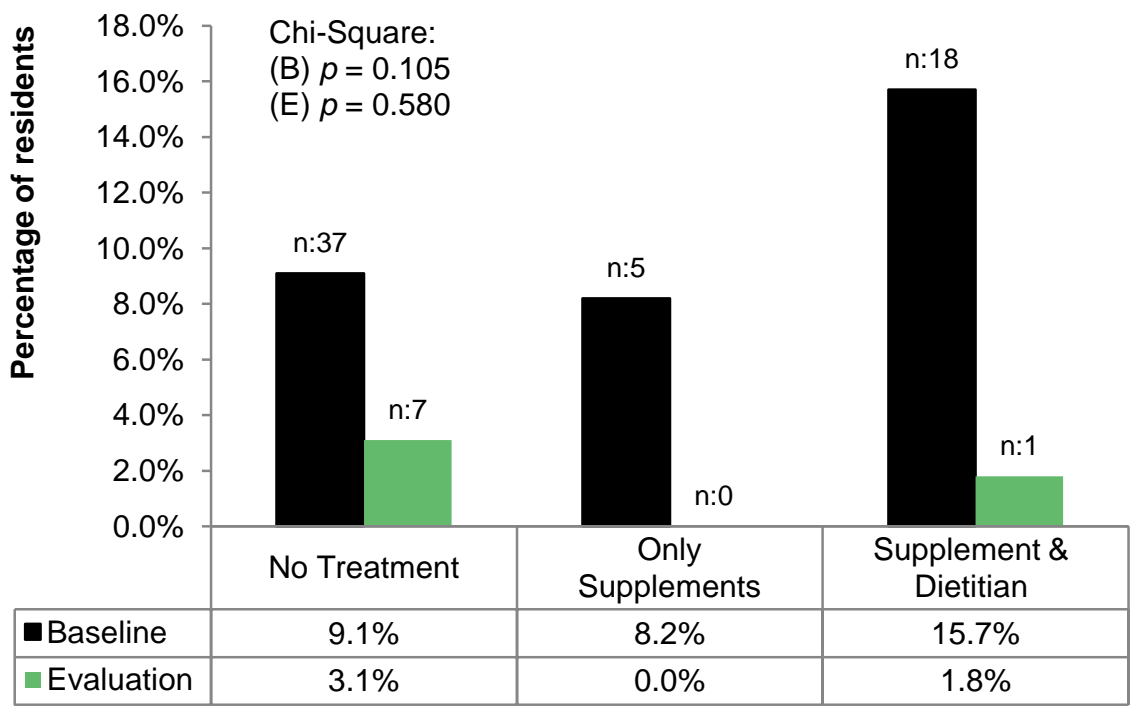

Fig. 10 Prevalence of pressure ulcers in "at risk" residents according to treatment.

\section{Discussion}

This service evaluation has shown FoU delivered by dietetic assistants positively impacts undernutrition outcome measures in care homes, through achieving significant improvements in weight, undernutrition and PU outcome measures that are comparable to published research. FoU was one of the first dietetic services to promote food based interventions (FB) [55], defined as "no treatment" in the results. No systematic review [63-66] has made specific conclusions regarding nutritional interventions for undernutrition in care homes [51], due to inconsistent and limited strong quality evidence for the impact of FB on outcomes [67]. However, analysis of the three "at risk" treatments clearly indicates FoU alone ("no treatment") has a positive impact on all outcomes, although no significant difference was identified between treatments for any objective (Figs. 5, 7, 10).

\subsection{Weight Change}

The rate of weight change significantly improved in "at risk" residents following FoU, with significant differences identified between undernutrition risk and rate of weight change at baseline and evaluation (Table 6, Fig. 4). This is in keeping with findings from the nutritional screening week (NSW) survey [7], where undernutrition risk was significantly related to weight change, accounting for $9.3 \%$ variability in weight.

In keeping with other FB studies [51, 68], FoU led to a significant weight improvement in "at risk" residents, with the greatest impact seen in high risk gaining $1.0 \mathrm{~kg}$ (Table 7), the same weight achievement as FoU's pilot open learning homes [50], but greater than other studies [17, 69]. In moderate risk, weight continued to decline but at a significantly lesser rate (Fig. 4, Tables 6 and 7). These findings suggest scaling up FB research to a regional service can achieve comparable weight outcomes. However, further analysis using data out of scope is recommended to determine if care plan implementation is an influencing factor on weight change according to undernutrition risk.

Although rate of weight change improved overall; age, gender, care category, dementia and treatment type had no significant influence (Table 7). This is in keeping with NSW [7], although Tamura [70] found gender and age to significantly influence weight. Causes of weight loss are multifunctional [1], with dysphagia, leaving $>25 \%$ meals, eating dependency and dining environment being significant modifiable 
factors associated with weight loss [70-72]. Building on this finding, FoU already incorporates these factors into the dietary assessment part of "MUST" [54] to assist staff to develop appropriate nutrition care plans.

Prior to FoU $49.3 \%$ residents lost weight, with $14.6 \%$ losing > 10\%; both proportions increased with undernutrition risk (Table 9), which is comparable to a London-based audit [73], where 51\% lost weight, 14\% lost $>10 \%$. In high risk residents there was a substantial $73.4 \%$ reduction in residents losing $>10 \%$ weight and $176 \%$ improvement in residents gaining > $10 \%$ weight (Table 9). A weight loss of $4-5 \%$ during one year significantly increases mortality [74, 75]. The amount of high risk residents losing $>5 \%$ weight declined by $60.3 \%$ (Table 9), further analysis using data out of scope is recommended to determine if weight influenced mortality.

\subsection{Undernutrition Prevalence}

Prevalence of undernutrition significantly reduced by $11 \%$, to $29.1 \%$ (Table 10 ); $60 \%$ of "at risk" were high risk, reducing to $54.7 \%$. Although overall reduction is similar to the pilot [50] (13.6\%), overall prevalence was lower (44\%-38\%) as anticipated, because in the pilot's baseline no resident was screened for undernutrition; a finding which is unlikely to be identified today because undernutrition awareness and screening has improved following national initiatives (Table 1). In keeping with other studies undernutrition was significantly impacted by age $[4,7,76,77]$, female gender [7, 77-79], care type $[7,77,80]$, although duration was only significant at baseline which is in contrast to published work where duration had no impact $[6,76,81]$.

At baseline local prevalence (32.7\%) was lower or comparable to other UK studies using "MUST" [7, 51, 73, 76, 80-85] (31.9\%-41.6\%). FoU's lower prevalence is encouraging, because the North has $73 \%$ higher risk of undernutrition [4], in addition the North-East is a deprived region [86] and undernutrition is associated with deprivation [25], although Parsons [84] identified that deprivation did not impact undernutrition in care homes, but transferability is questionable being limited to one geographical region.

Comparing FoU's impact (Table 10, Fig. 6) on undernutrition is limited because other UK studies using "MUST" [17, 51, 85, 87] did not report on prevalence change; and foreign studies [61, 68, 69, 88 -91] used alternative outcomes or non-comparable nutritional screening tools (NST). Similar studies [83, 92] implementing education and care pathways identified both a reduction [92] and no impact [83] in prevalence.

FoU uses "MUST" [53]; recommended by many UK organizations [7], simple, acceptable and quick to use [93], with very good inter-observer reliability and fair validity in care homes [53, 94, 95], used by $96 \%$ care homes [7]. FoU was the first UK service to systematically implement "MUST" into care homes, through adapting the layout for simplicity [54, 96], which independent research has identified to be simpler, quicker, more accurate and preferred to original "MUST" [57].

"MUST" completion significantly improved (76.3\%-98.7\%), reflective of other studies following training [51, 85, 92]. Accuracy not completion should be key to audits [96, 97]. Inter-observer agreement of undernutrition risk identified by the care home's NST and FoU "MUST" was fair (K:0.33), with $60 \%$ overall agreement. Historically, homes used varied NST, but original "MUST" has become the prominent NST, reflective of the cultural change nationally [7]. Unfortunately no comparison for accuracy is available at evaluation, although accuracy is incorporated into FoU's reaccreditation (Appendix 1).

Training is key to implementing "MUST" [28, 96, 97], supported by interventions identified by staff to help overcome barriers [28, 98]. Since conception FoU has embedded these principles through adapting "MUST" [54], developing practical resources [99] and training to empower and skill staff $[58,100]$. 


\subsection{Pressure Ulcers}

Overall PU development significantly declined 51\%, with prevalence significantly increasing with severity of undernutrition risk, but following FoU there was no difference between risks. PU development is multifactorial [101], but significantly associated with undernutrition [14, 102]. Accounting for 33\% costs associated with PU103, nutritional intervention is one of the most cost effective strategies for PU [104]. Undernutrition is a reversible PU risk factor [102], so early identification and treatment is essential [102, 104]. Through screening and interventions FoU was associated with a $68 \%$ reduction of PU in "at risk" residents (Fig. 9). Although there is a lack of strong evidence between undernutrition and PU [105, 106], these findings are comparable with predicted reductions when treated with ONS [105, 107].

Increased odds of PU are identified with increased severity of undernutrition $[14,108]$, or $>5 \%$ weight loss [102]. FoU interventions reduced the odds of developing PU, with greatest odds seen in moderate risk, indicating a 78\% PU reduction (Table 13). This is in keeping with other studies where improved nutrition knowledge [108], undernutrition screening [108], ONS treatment [105] have reduced PU odds.

\subsection{Strengths and Limitations}

This is the first large scale service evaluation on undernutrition for care homes in England. However, as a retrospective service evaluation the limitations were the constraints on data collection to evaluate outcomes compared to research, such as usage of health resources. While FoU demonstrated the potential to reduce possible harms, such as PU, it was out of scope to evaluate the impact on other potential harms such as infections or falls, which reduce with improved nutritional status [13]. Although formal cost analysis is beyond the scope of this evaluation, these findings suggest a dietetic service delivered by dietetic assistants has the potential to be effective in achieving comparable outcomes when evaluated alongside other treatment options. Data collection was dependant on the data quality in resident records, and once data collection was established scope to incorporate new measures such as antibiotic usage, falls was limited. Changes in rate of weight change were small, which may be due to fluid; but FoU's true impact could be misrepresented using rate, because the magnitude of weight increase declines over time, as identified by Lorefalt [61]. Considering this, weight was also analyzed as actual and percentage weight change to compare alongside literature. Finally historical database issues meant missing data could not be sought.

Nevertheless, the strengths of being a large scale pragmatic service evaluation undertaken over 13 years and incorporating an uncontrolled generic care home population, suggest these findings are reflective of everyday practices.

\subsection{Recommendations}

As identified further analysis is required using out of scope data, in addition to previous recommendations comparing weight data alongside NSW [7] findings, further analysis of "at risk" treatments, training outcomes, and cost effectiveness of FoU are recommended.

Elia [29] advocated "it is imperative national policy ensures undernutrition detection and treatment are embedded in routine care", through training, integrated accurate systems of recording and auditing undernutrition management $[7-10,16,29,31,35$, 40, 73, 101]. Although FoU delivers on these requirements, it is paramount FoU continues to evidence itself continually in terms of quality improvement. FoU as a dietetic example of a preventative public health initiative can impact the Five-Year Plan agenda [109] and all domains of the NHS Outcome framework [110]. To evidence this, future evaluation on FoU's sustainability needs to analyze the structure, process indicators of undernutrition care; collected annually during 
reaccreditation (Appendix 1). Such evaluation links to Donabedian's quality theory [111], where outcome is dependent on structural and process indicators. While some [73, 101, 112-114] identified such evaluation improved nutritional care, others [71] found it failed to predict undernutrition. To evaluate resident outcomes and benchmark homes locally and nationally, it is recommended that BAPEN's nutritional care tool [115] is explored to determine if it can be incorporated into FoU's quality standard (Appendix 1), thereby requiring homes to complete annually during reaccreditation.

\subsection{Conclusion}

FoU is multifaceted, delivering multi-disciplinary whole home training, alongside tailored support, practical resources and annual reaccreditation against quality standards (Appendix 1) which are linked to Local Authority funding incentives; all of which have been identified to be key to undernutrition management (Table 1) [28, 73, 96, 101]. Although numerous studies have shown improvements in undernutrition following training $[61,85,87,89,92$, 97, 116], FoU's multifaceted approach to undernutrition is identified to be more effective than a single approach such as training or FB [61, 117]. This paper has demonstrated that FoU delivered by dietetic assistants is a significantly effective approach for dietetic services to improve the management of undernutrition in care homes.

\section{Conflict of Interest and Funding}

RM declares no conflicts of interest.

$\mathrm{RM}$ is an MSc student at Newcastle University and the senior specialist dietitian for Focus on Undernutrition at County Durham and Darlington NHS Foundation Trust. This evaluation was undertaken as a student project as part of the National Institute for Health Research (NHIR) Clinical Academic Training Programme.

\section{Acknowledgements}

RM thanks God for the key people who have supported her through this journey, including:

Mike Hartley: whom this dissertation would never have been completed without his expert skills and time during the stages of redeveloping, merging databases and data cleaning.

Focus on Undernutrition team: the dietetic assistants who have spent many weeks inputting data onto the FoU database, and every FoU team member who has supported me during this whole journey. This dissertation is for you all, to prove what an amazing service you all provide.

Newcastle university staff: Lisa Robinson: for her guidance and encouragement as university mentor, and Matthew Linsley for his guidance on statistics.

Jennie Winnard: for suggesting this dissertation topic, being a workplace mentor and rearranging clinical workloads to support the undertaking this MSc.

Colleagues: who have provided guidance on proof reading.

Finally and most importantly, to James, Hannah, Rebekah and dad: for your priceless support and encouragement during these last two years.

\section{References}

[1] Dyck, M. J., and Schumacher, J. R. 2011. "Evidence-Based Practices for the Prevention of Weight Loss in Nursing Home Residents." Journal of Gerontological Nursing 37 (3): 22-33.

[2] Elia, M. 2015. "The Cost of Malnutrition in England and Potential Cost Savings from Nutritional Interventions (Short Version): A Report on the Cost of Disease-Related Malnutrition in England and a Budget Impact Analysis of Implementing the NICE Clinical Guidelines/Quality Standards on Nutritional Support in Adults." Written on behalf of the Malnutrition Action Group of the British Association for Parenteral and Enteral Nutrition and the National Institute for Health Research Southampton Biomedical Research Centre. ISBN: 978-1-899467-03-3. Available from: http://www.bapen.org.uk/pdfs/economic-report-short.pdf (Accessed 19 July 2016). 
[3] Elia, M., and Russell, C. 2009. Combating Malnutrition: Recommendations for Action. A report from the Advisory Group on Malnutrition, led by BAPEN. Redditch, BAPEN. Available from: http://www.bapen.org.uk/pdfs/reports/advisory_group_re port.pdf (Accessed 19 July 2016).

[4] Elia, M., and Stratton, R. J. 2005. "Geographical Inequalities in Nutrient Status and Risk of Malnutrition among English People Aged 65 Years and Older." Nutrition 21: 1100-6.

[5] Stratton, R. J. 2006. "Deprivation Linked to Malnutrition Risk and Mortality in Hospital." British Journal of Nutrition 96: 870-6.

[6] Russell, C., and Elia, M. 2011. Nutrition Screening Survey in the UK and Republic of Ireland in 2010; in Hospital, Care Homes and Mental Health Units. A report by the British Association for Parenteral and Enteral Nutrition (BAPEN). ISBN: 978-1-899467-81-5. Available at: http://www.bapen.org.uk/pdfs/nsw/nsw-2011-report.pdf (Accessed: 19 July 2016).

[7] Russell, C., and Elia, M. 2015. Nutrition Screening Survey in Care Homes in England. A Reported Based on the Amalgamated Data from the Four Nutritional Screening Week Surveys Undertaken by BAPEN in 2007, 2008, 2010 and 2011. A report by the British Association for Parenteral and Enteral Nutrition (BAPEN). ISBN: 978-1-899467-97-1. Available at: http://www.bapen.org.uk/pdfs/nsw/care-homes/care-hom es-england.pdf (Accessed: 19 July 2016).

[8] NHS England. Guidance-Commissioning Excellent Nutrition and Hydration 2015-2018. Available from: https://www.england.nhs.uk/wp-content/uploads/2015/10/ nut-hyd-guid.pdf (Accessed 22 July 2016).

[9] Malnutrition Task Force. 2013. Prevention and Early Interventions of Malnutrition in Later Life. Best Practice Principles and Implementation Guide-Care Homes. Available from: http://www.malnutritiontaskforce.org.uk/wp-content/uplo ads/2014/07/CH-Prevention_Early_Intervention_Of_Mal nutrition_in_Later_Life_Care_Home.pdf (Accessed 22 July 2016).

[10] National Institute for Health and Clinical Excellence. 2014. Quality Standard for Nutrition Support in Adults, NICE Quality Standard 24. 2012. Available at: https://www.nice.org.uk/guidance/qs24/resources/nutritio n-support-in-adults-2098545777349 (Accessed 22 July 2016).

[11] Flacker, J. M., and Kiely, D. K. 1998. "A Practical Approach to Identifying Mortality Related Factors in Established Long Term Care Residents." Journal of the American Geriatrics Society 46: 1012-5.
[12] Sullivan, D. H., Morley, J. E., Johnson, L. E., et al. 2002. "The GAIN (Geriatric Anorexia Nutrition) Registry: The Impact of Appetite and Weight on Mortality in a Long Term Care Population." Journal of Nutrition, Health and Aging 6: 275-81.

[13] Stratton, R. J., Green, C., and Elia, M. 2003. Disease Related Malnutrition: An Evidence-Based Approach to Treatment. Oxford: CABI.

[14] Banks, M., Bauer, J., Graves, N., et al. 2010. "Malnutrition and Pressure Ulcer Risk in Adults in Australian Health Care Facilities." Nutrition 26: 896-901.

[15] Crogan, N. L., and Pasvogel, A. 2003. "The Influence of Protein Energy Malnutrition on Quality of Life in Nursing Homes." Journal of Gerontology 58: 159-64.

[16] Arvanitakis, M., Beck, A., Coppens, P., et al. 2008. "Nutrition in Care Homes and Home Care: How to Implement Adequate Strategies (Report from the Brussels Forum 22-23 November 2007)." Clinical Nutrition 27: 481-8.

[17] Parsons, E. L., Stratton, R. J., Cawood, A., et al. 2016. "Oral Nutritional Supplements in a Randomised Trial Are More Effective Than Dietary Advice at Improving Quality of Life in Malnourished Care Home Residents." Clinical Nutrition [Preprint].

[18] Burger, S. G., Kayser-Jones, J., and Prince, J. 2000. Malnutrition and Dehydration in Nursing Homes: Key Issues in Prevention and Treatment. Available from: http://www.commonwealthfund.org/usr_doc/burger_mal_ 386.pdf (Accessed 22 July 2016).

[19] Guest, J. F., Panca, M., Baeyens, J. P., et al. 2011. "Health Economic Impact of Managing Patients Following a Community Based Diagnosis of Malnutrition in the UK." Clinical Nutrition 30: 422-9.

[20] Meijers, J. M. M., Halfens, R. J. G., Wilson, L., et al. 2012. "Estimating the Costs Associated with Malnutrition in Dutch Care Homes." Clinical Nutrition 31: 65-8.

[21] European Nutrition for Health Alliance. 2006. Malnutrition among Older People in the Community Policy Recommendations for Change. Available from: http://www.european-nutrition.org/images/uploads/pdf_p df_37.pdf (Accessed 22 July 2016).

[22] Elia, M., Zellipour, L., and Stratton, R. J. 2005. "To Screen or Not to Screen for Adult Malnutrition?" Clinical Nutrition 24: 867-84.

[23] National Institute for Health and Clinical Excellence. 2006. Clinical Guideline 32: Nutritional Support in Adults: Oral Nutritional Support, Enteral Tube Feeding and Parenteral Nutrition." London: NICE. Available at: https://www.nice.org.uk/guidance/cg32/resources/nutritio n-support-for-adults-oral-nutrition-support-enteral-tube-f eeding-and-parenteral-nutrition-975383198917. (Accessed: 22 July 2016). 
[24] Wilson, L. 2013. "A Review and Summary of the Impact of Malnutrition in Older People and the Reported Costs and Benefits of Interventions. On Behalf of the Malnutrition Task Force." Available at: http://www.malnutritiontaskforce.org.uk/wp-content/uplo ads/2014/07/General-Costs_and_Benefits_Report_June_2 013.pdf (Accessed: 22 July 2016).

[25] Stratton, R. J. 2007. "Pennington Lecture-Malnutrition: Another Health Inequality?" Proceedings of the Nutrition Society 66: 522-9.

[26] National Institute of Health and Clinical Excellence. 2011. Nutrition Support in Adults (32) Cost Saving Guidance. Available at: https://www.nice.org.uk/guidance/cg32/resources/costing -report-194884669 (Accessed: 22 July 2016).

[27] Volkert, D., Saeglitz, C., and Gueldenzoph, H., et al. 2010. "Undiagnosed Malnutrition and Nutrition Related Problems in Geriatric Patients." Journal of Nutrition Health Ageing 14: 387-92.

[28] Green, S. M., James, E. P., Latter, S., Sutcliffe, M., and Fader, M. J. 2014. "Barriers and Facilitators to Screening for Malnutrition by Community Nurses: A Qualitative Study." Journal of Human Nutrition and Dietetics 27: 88-95.

[29] Elia, M., Russell, C. A., and Stratton, R. J. 2010. "The Skeleton in the Closet: Malnutrition in the Community. Malnutrition in the UK: Policies to Address the Problem." Proceedings of the Nutrition Society 69: 470-6.

[30] Lecko, C. 2013. Patient Safety and Nutrition and Hydration in the Elderly. A Health Foundation Report. Available from: http://patientsafety.health.org.uk/sites/default/files/resour ces/patient_safety_and_nutrition_and_hydration_in_the_ elderly.pdf (Accessed 22 July 2016).

[31] The Patients Association. 2015. Managing Adult Malnutrition in the Community: Summary Report. A Spotlight on Information, Help and Support Available for Patients and Carers in England." Available from: http://www.patients-association.org.uk/wp-content/uploa ds/2015/11/managing-adult-malnutrition-in-the-communi ty-summary-nov2015.pdf (Accessed 22 July 2016).

[32] Care Quality Commission. 2012. Time to Listen in Care Homes: Dignity and Nutrition Inspection Programme 2012. Available at: http://www.cqc.org.uk/Sites/default/files/documents/time _to_listen_-_care_homes_main_report_tag.pdf. (Accessed: 22 July 2016).

[33] Care Quality Commission. 2010. Guidance about Compliance Essential Standards of Quality and Safety. London: Care Quality Commission. Available from: http://www.cqc.org.uk/content/regulation-14-meeting-nut ritional-and-hydration-needs\#full-regulation. (Accessed:
22 July 2016).

[34] British Association for Parenteral and Enteral Nutrition (BAPEN). 2014. Malnutrition Matters: A Commitment to Act. Redditch: BAPEN. Available from: http://www.bapen.org.uk/pdfs/malnutrition-matters-a-co mmitment-to-act.pdf (Accessed 22 July 2016).

[35] Brotherton, A., Stroud, M., and Simmonds, N. 2012. Malnutrition Matters: Meeting Quality Standards in Nutritional Care. A Toolkit for Clinical Commissioning Groups and Providers in England. Second edition. Redditch: BAPEN. Available from: http://www.bapen.org.uk/pdfs/bapen_pubs/bapen-toolkitfor-commissioners-and-providers.pdf (Accessed: 22 July 2016).

[36] Malnutrition Pathway. 2012. Managing Adult Malnutrition in the Community, Including a Pathway for the Appropriate Use of Oral Nutritional Supplements. http://Malnutritionpathway.co.uk/downloads/Managing_ Malnutrition.pdf (Accessed 22 July 2016).

[37] National Prescribing Centre. 2012. Prescribing of Adult Oral Nutritional Supplements (ONS): Guiding Principles for Improving the Systems and Processes for ONS Use. Available at: http://www.npc.nhs.uk/quality/ONS/resources/borderline _substances_final.pdf (Accessed: 28 November 2014).

[38] PRESCqipp. 2014. Guidelines for the Appropriate Prescribing of Oral Nutrition (ONS) for Adults in Primary Care. Available from: https://www.prescqipp.info/resources/send/106-ons-guide lines/1348-onsg (Accessed 22 July 2016).

[39] British Dietetic Association. 2012. The Nutrition and Hydration Digest: Improving Outcomes through Food and Beverage Services. https://www.bda.uk.com/publications/professional/Nutriti onHydrationDigest.pdf (Accessed 22 July 2016).

[40] Council for Europe. 2009. 10 Key Characteristics of Good Nutritional Care and Associated Factsheets. http://www.nrls.npsa.nhs.uk/resources/?entryid45=59865 (Accessed 22 July 2016).

[41] Institute for Innovation and Improvement. 2008. High Impact Actions: Keeping Nourished, Getting Better. http://www.institute.nhs.uk/building_capability/hia_supp orting_info/keeping_nourished_getting_better.html (Accessed 22 July 2016).

[42] Department of Health. 2010. Essence of Care Benchmarks for Food and Drink. Leeds: Department of Health. Available from: https://www.gov.uk/government/uploads/system/uploads/ attachment_data/file/216696/dh_125313.pdf (Accessed 22 July 2016).

[43] National Association for Care Caterers. 2010. Мenu Planning and Special Diets in Care Homes. Available 
from:

http://www.thenacc.co.uk/shop/product/Menu+Planning+ and+Special+Diets+in+Care+Homes (Accessed 22 July 2016).

[44] Caroline Walker Trust. 2004. Eating Well for Older People. Practical and Nutritional Guidelines for Food in Residential and Nursing Homes and for Community Meals. Second edition. ISBN 189782018 6. Available from:

http://www.cwt.org.uk/wp-content/uploads/2014/07/Olde rPeople.pdf (Accessed 22 July 2016).

[45] Department of Health and Other Stakeholders. 2007. Improving Nutritional Care: Nutrition Action Plan. Leeds: Department of Health. Available from: http://webarchive.nationalarchives.gov.uk/201301071053 54/http://www.dh.gov.uk/prod_consum_dh/groups/dh_di gitalassets/@dh/@en/documents/digitalasset/dh_079932. pdf (Accessed 22 July 2016).

[46] British Association for Parenteral and Enteral Nutrition (BAPEN). Nutritional Screening Week (2006-2011). Available from: http://www.bapen.org.uk/screening-and-must/nutrition-sc reening-week/nsw-overview (Accessed 27 July 2016).

[47] Nutrition and Hydration Week. Available from: https://nutritionandhydrationweek.co.uk/ (Accessed 22 July 2016).

[48] British Dietetic Association. Mind the Gap. Available from: http://www.mindthehungergap.com/ (Accessed 22 July 2016).

[49] Royal College of Nursing. 2009. Nutrition Now: Enhancing nutritional care. Available from: https://www2.rcn.org.uk/_data/assets/pdf_file/0006/187 989/003284.pdf (Accessed 22 July 2016).

[50] Masters, R. 2003. "Focus on Food": A Strategic Approach to Undernutrition in Nursing Homes throughout County Durham, Darlington and Teesside Executive Summary. Available from: http://www.focusonundernutrition.co.uk/repository/docu ments/editorfiles/evidence/Focus\%20on\%20Food\%20pil ot\%20report/Executive\%20summary\%20of\%20FoF\%20 pilot.pdf (Accessed 22 July 2016).

[51] Stow, R., Ives, N., Smith, C., et al. 2015. "A Cluster Randomised Feasibility Trial Evaluating Nutritional Interventions in the Treatment of Malnutrition in Care Homes." Trials 16: 433-54. doi: 10.1186/s13063-015-0952-2.

[52] Food First Team. http://www.sept.nhs.uk/our-services/bedfordshire-and-lut on/bedfordshire-community-health-services/adults/nutriti on-dietetics/food-first/ (Accessed 22 July 2016).

[53] Elia, M. 2003. The "MUST" Report. Nutritional Screening for Adults: A Multidisciplinary Responsibility.
Development and Use of the "Malnutrition Universal Screening Tool" ("MUST") for Adults. A report by the Malnutrition Advisory Group of the British Association for Parenteral and Enteral Nutrition. Redditch: BAPEN.

[54] Focus on Undernutrition's "MUST". Available from: http://www.focusonundernutrition.co.uk/repository/docu ments/editorfiles/Focus\%20resources/MUST/MUST_(Nu tritional_2pp_A4)_(Comm\%20ONS)\%20Watermark_(Fo U1.001.1.3).pdf (Accessed 22 July 2016).

[55] Focus on Undernutrition's Care Pathway for the Treatment of Undernutrition in Adults. Available from: http://www.focusonundernutrition.co.uk/repository/docu ments/editorfiles/howto/Care_Pathway_for_screening.pdf (Accessed 22 July 2016).

[56] Focus on Undernutrition's Care Pathway for the Prescribing of Oral Nutritional Supplements in Adults. Available from: http://www.focusonundernutrition.co.uk/repository/docu ments/editorfiles/pdfs/CDDFT\%20FOU\%20Care\%20Pat hway\%20-\%20final\%2017\%202014\%20on\%20website.p df (Accessed 2 August 2016).

[57] Ralph, A. F., Cawood, A. L., Walters, E., et al. 2010. "Evaluation of an Alternative Set of Supporting Materials for 'MUST' across the Hospital and Community Setting.' Proceedings of the Nutrition Society 69: (OCE7), E571.

[58] Focus on Undernutrition in Care Homes. Available from: http://www.focusonundernutrition.co.uk/focus-services/c are-homes-for-older-people (Accessed 22 July 2016).

[59] Focus on Undernutrition Feedback. Available from: http://www.focusonundernutrition.co.uk/about-focus/focu s-feedback (Accessed 22 July 2016).

[60] Malnutrition Task Force, link for Focus on Undernutrition. Available from: http://www.malnutritiontaskforce.org.uk/wp-content/uplo ads/2014/07/CH-Focus_on_Undernutrition_County_Durh am.pdf (Accessed 22 July 2016).

[61] Lorefalt, B., and Wilhelmsson, S. 2012. "A Multifaceted Intervention Model can Give a Lasting Improvement of Older Peoples' Nutritional Status." Journal of Nutrition, Health \& Aging 16: 378-82.

[62] Petrie, A., and Sabin, C. 2010. Medical Statistics at a Glance. Blackwell Science Ltd: Oxford. ISBN: 0-632-05075-6.

[63] Baldwin, C., and Weekes, C. E. 2012. "Dietary Advice with or without Oral Nutritional Supplements for Disease-Related Malnutrition in Adults (Review)." Cochrane Database Systematic Review 9.

[64] Milne, A. C., Potter, J., and Vivanti, A. 2009. "Protein and Energy Supplementation in Elderly People at Risk from Malnutrition (Review)." Cochrane Database Systematic Review 2.

[65] Kimber, K., Gibbs, M., Weekes, C. E., et al. 2015. 
"Supportive Interventions for Enhancing Dietary Intake in Malnourished or Nutritionally At-Risk Adults: A Systematic Review of Nonrandomised Studies." Journal of Human Nutrition and Dietetics 28: 517-45.

[66] Gibbs, M., Baldwin, C., and Weekes, C. E. 2012. "Supportive Interventions for Enhancing Dietary Intake in Malnourished or Nutritionally at risk Adults (Protocol)." Cochrane Database Systematic Review 5.

[67] Baldwin, C., and Weekes, C. E. 2012. "Dietary Counselling with or without Oral Nutritional Supplements in the Management of Malnourished Patients: A Systematic Review and Meta-Analysis of Randomised Controlled Trials." Journal of Human Nutrition and Dietetics 25: 411-26.

[68] Leslie, W. S., Woodward. M., Lean, M. E. J., et al. 2012. "Improving the Dietary Intake of Undernourished Older People in Residential Care Homes Using an Energy-Enriching Food Approach: A Cluster Randomised Controlled Study." Journal of Human Nutrition and Dietetics 26: 387-94.

[69] Odlund Olin, A., Armyr, I., Soop, M., et al. 2003. "Energy-Dense Meals Improve Energy Intakes in Elderly Residents in a Nursing." Clinical Nutrition 22: 125-31.

[70] Tamura, B. K., Bell, C. L., Masaki, M. D., et al. 2013. "Factors Associated with Weight Loss, Low BMI and Malnutrition among Nursing Home Patients: A Systematic Review of the Literature." Journal of American Medical Directors 4 (9): 649-55.

[71] Bonaccorsi, G., Collini, F., Castagnoli, M., et al. 2015. "A Cross Sectional Survey to Investigate the Quality of Care in Tuscan (Italy) Nursing Homes: The Structural, Process and Outcome Indicators of Nutritional Care." BioMed Central Health Service Research 2015: 1-10. doi: 10.1186/s12913-015-0881-5.

[72] Beck. A. M. 2015. "Weight Loss, Mortality and Associated Potentially Modifiable Nutritional Risk Factors among nursing Home Residents-A Danish Follow-Up Study." Journal of Nutrition, Health \& Aging 19: 96-100.

[73] Weekes, C. E. 2014. "The Lambeth and Southwark Action on Malnutrition Project: Auditing Compliance with National Nutritional Care Guidelines in Community Healthcare Settings-Older Peoples' Care Homes." Unpublished.

[74] Alibhai , S. M., Greenwood, C., and Payette, H. 2005. "An Approach to the Management of Unintentional Weight Loss in Elderly People." Canadian Medical Association Journal 172: 773-80.

[75] Diekmann, R., Winning, K., Uter, W., et al. 2013. "Screening for Malnutrition among Nursing Home Residents-A Comparative Analysis of the Mini Nutritional Assessment, the Nutritional Risk Screening and the "Malnutrition Universal Screening Tool'." The Journal of Nutrition, Health and Aging 17: 326-31.

[76] Ralph, A. F., Cawood, A. L., Elia, M., et al. 2010. "Factors Affecting Malnutrition Prevalence in Care Homes in Dorset, UK." Clinical Nutrition Supplement 5: 171.

[77] Van Nie-Visser, N. C., Meijers, J., Schols, J., et al. 2014. "Which Characteristics of Nursing Home Residents Influence Differences in Malnutrition Prevalence? An International Comparison of the Netherlands, Germany and Austria." British Journal of Nutrition 11: 1129-36.

[78] Perissinotto, E., Schutz, T., Normal, K., et al. 2002. "Anthropometric Measurements in the Elderly, Age and Gender Differences." British Journal of Nutrition 87: 177-86.

[79] Meijers, J., Schols, J., Van Bokhorst-de Van der Schueren, M., et al. 2009. "Malnutrition Prevalence in the Netherlands: Results of the Annual Dutch Malnutrition Prevalence Measurement of Care Problems." British Journal of Nutrition 101: 417-23.

[80] Norris, E. S. A., Shelton, F., and Hetherington, M. M. 2011. "Nutritional Screening of Older Adults Living in Care Homes." e-Journal of Clinical Nutrition and Metabolism 6: e106-8. Available from: doi:10.01016/j.eclnm.2011.04.002 (Accessed 25 June 2016).

[81] Parsons, E. L., Cawood, A. L., Warwick, H., et al. 2010. "Malnutrition Risk Varies according to Nutrition Interventions in Care Homes." Clinical Nutrition Supplement 5: 161.

[82] Cawood, A. L., Smith, A., Dalrymple-Smith., J., et al. 2012. "Prevalence of Malnutrition and Use of Nutritional Support in Peterborough Primary Care Trust." Journal of Human Nutrition and Dietetics 21: 384.

[83] Forbes, C., Temple, A., Romano, V., et al. "Review of the Impact of Food First Advice, Training and Support on the Nutritional Outcomes of Residents in Long Term Care." Unpublished abstract.

[84] Parsons, E. L., Stratton, R. J., and Elia, M. 2010. "Deprivation Is Not Associated with Malnutrition Risk in Care Homes in Hampshire, UK." Clinical Nutrition Supplement 5: 170

[85] Cawood, A., Smith, A., Pickles, S., et al. 2009. "Effectiveness of Implementing 'MUST' into Care Homes within Peterborough Primary Care Trust, England." Clinical Nutrition Supplements 2: 81.

[86] Department for Local Communities and Local Government. 2015. The English Indices of Deprivation 2015 Statistical Release. ISBN: 978-1-4098-4688-8. Available from: https://www.gov.uk/government/uploads/system/uploads/ attachment_data/file/465791/English_Indices_of_Depriva 
tion_2015_-_Statistical_Release.pdf (Accessed 26 July 2016).

[87] Lee, R., and Scott, F. 2009. "Competent to Care. A Train-the-Trainer Method of Teaching as a Way of Implementing the Correct Use of the 'Malnutrition Universal Screening Tool' in Norfolk: Is It Effective?" Proceedings of the Nutrition Society 68: 300-5.

[88] Gaskill, D., Isenring, E. A., and Black, L. J. 2009. "Maintaining Nutrition in Aged Care Residents with a Train-the-Trainer Intervention and Nutrition Coordinator." The Journal of Nutrition, Health \& Aging 13: 913-7.

[89] Wikby, K., Ek, A. C., and Christensson, L. 2009. "Implementation of a Nutritional Programme in Elderly People Admitted to Resident Homes." Scandinavian Journal of Caring Science 23: 421-30.

[90] Beck, A. M., Ovesen, L., and Schroll, M. 2002. "Home-Made Oral Supplement as Nutritional Support of Old Nursing Home Residents, Who Are Undernourished or at Risk of Undernutrition Base on the MNA. A Pilot Trial." Aging Clinical and Experimental Research 14: 212-5.

[91] Simmons, S. F., Keeler, E., An, R., et al. 2015. "Cost-Effectiveness of Nutrition Intervention in Long-Term Care." Journal American Geriatrics Society 63: 2308-16.

[92] Molyneux, A., Hogan, H., and Burns, A. 2010. "Implementation of a Nursing Home Care Management Pathway Developed to Facilitate the Use of the 'Malnutrition Universal Screening Tool'." Proceedings of the Nutrition Society 69 (OCE7): E550.

[93] Elia, M., and Stratton, R. J. 2011. "Considerations for Screening Tool Selection and Role of Predictive and Concurrent Validity." Current Opinion in Clinical Nutrition and Metabolic Care 14: 425-33.

[94] Isenring, E. A., Banks, M., Ferguson, M., et al. 2012. "Beyond Malnutrition Screening: Appropriate Methods to Guide Nutrition Care for Aged Care Residents." Journal of the Academy of Nutrition and Dietetics 112: 376-81.

[95] Poulia, K. A., Yannakoulia, M., Karageorgou, D., et al. 2012. "Evaluation of the Efficacy of Six Nutritional Screening Tools to Predict Malnutrition in the Elderly." Clinical Nutrition 31: 378-85.

[96] Smith, A. 2014. "Potential Barriers to Effective 'MUST' Implementation." Nutrition 2014: 28-31.

[97] Farrer, K., Donaldson, E., Blackett, B., et al. 2014. "Nutritional Screening of Elderly Patients: A Health Improvement Approach to Practice." Journal of Human Nutrition and Dietetics 27: 184-91.

[98] Baker, R., Camosso-Stefinovic, J., Gillies, C., et al. 2010. "Tailored Interventions to Overcome Identified Barriers to Change: Effects on Professional Practice and Healthcare Outcomes (Review)." Cochrane Database
Systematic Review 3.

[99] Focus on Undernutrition's resources. Available from: http://www.focusonundernutrition.co.uk/focus-resources (Accessed 28 July 2016).

[100] Focus on Undernutrition's Training. Available from: http://www.focusonundernutrition.co.uk/focus-training/fo cus-training-e-learning (Accessed 28 July 2016).

[101] Parsons, E. L., Baldwin, C., Fitzpatrick, J., et al. 2013. "Designing a National Clinical Audit of Nutritional Care in Health and Social Care Settings: Considerations and Future Directions." Proceedings of the Nutrition Society 72: 251-60.

[102] Shahin, E. S. M., Meijers, J. M. M., Schols, J. M. G. A., et al. 2010. "The Relationship between Malnutrition Parameters and Pressure Ulcers in Hospitals and Nursing Homes." Nutrition 26: 886-9.

[103] Banks, M. D., Graves, N., Bauer, J. D., et al. 2010. “The Costs Arising from Pressure Ulcers Attributable to Malnutrition." Clinical Nutrition 29: 108-86.

[104] Hisashige, A., and Takehiko, O. 2012. "Cost-Effectiveness of Nutritional Interventions on Healing of Pressure Ulcers." Clinical Nutrition 31: 868-74.

[105] Stratton, R. J., Ek, A. C., Engfer, M., et al. 2005. "Enteral Nutritional Support in Prevention and Treatment of Pressure Ulcers: A Systematic Review and Meta-Analysis." Ageing Research Reviews 4: 422-50.

[106] Laner, G., Schloemer, G., Knerr,A., et al. 2005. "Nutrition for Preventing and Treating Pressure Ulcers (Review)." Cochrane Database Systematic Review 4.

[107] Cawood, A. L., Green, C., and Stratton, R. J. 2010. “The Budget Impact of Using Oral Nutritional Supplements in Older Community Patients at High Risk of Malnutrition in England." Proceedings of the Nutrition Society 69: (OCE7): E544.

[108] Iizaka, S., Okuwa, M., and Sugama, J. 2010. "The Impact of Malnutrition and Nutrition-Related Factors on the Development and Severity of Pressure Ulcers in Older Patients Receiving Home Care." Clinical Nutrition 29: 47-53.

[109] NHS England. 2014. Five Year Forward View. Available from:

https://www.england.nhs.uk/wp-content/uploads/2014/10/ 5yfv-web.pdf (Accessed 2 August 2016).

[110] Department of Health. 2014. The NHS Outcomes Framework 2015-2016. Available from: https://www.gov.uk/government/uploads/system/uploads/ attachment_data/file/385749/NHS_Outcomes_Framewor k.pdf (Accessed 2 August 2016).

[111] Donabedian, A. 1988. "The Quality of Care: How Can It Be Assessed?" Journal American Medical Association 260: 1743-8.

[112] Van Nie, N. C., Meijers, J. M. M., Schols, J. M. G. A., et 
al. 2014. "Do Structural Quality Indicators of Nutritional Care Influence Malnutrition Prevalence in Dutch, German and Austrian Nursing Homes?" Nutrition 30: 1384-90.

[113] Meijers, J. M. M., Tan, F., Schols, J. M. G. A., et al. 2014. "Nutritional Care: Do Process and Structure Indicators Influence Malnutrition Prevalence over Time?" Clinical Nutrition 33: 459-65.

[114] Schonherr, S., Halfens, R. J. G., and Meijers, J. M. M. 2012. "Structural and Process Indicators of Nutritional Care: A Comparison between Austrian Hospital and Nursing Homes." Nutrition 28: 868-73.

[115] British Association for Parenteral and Enteral Nutrition
(BAPEN). Nutritional Care Tool. Available from: http://www.data.bapen.org.uk/ (Accessed 28 July 2016).

[116] Kennelly, S., Kennedy, N. P., Rughoobur, G. F., et al. 2010. "An Evaluation of a Community Dietetics Intervention on the Management of Malnutrition for Healthcare Professionals." Journal of Nutrition and Dietetics 23: 567-74.

[117] Beck, A. M., Damkjaer, K., and Sorbye, L. W. 2010. "Physical and Social Functional Abilities Seem to Be Maintained by a Multifaceted Randomised Control Nutritional Intervention among Old (> 65 Years) Danish Nursing Home Residents." Archives of Gerontology and Geriatrics 50: 531-5. 


\section{Appendix 1: Quality standard used during reaccreditation}

\section{Focus on Undernutrition care home reaccreditation audit}

\begin{tabular}{|l|l|}
\hline Date completed & \\
\hline Year & baseline/evaluation/1 year/2 years/3 years/4 years/5 years \\
\hline
\end{tabular}

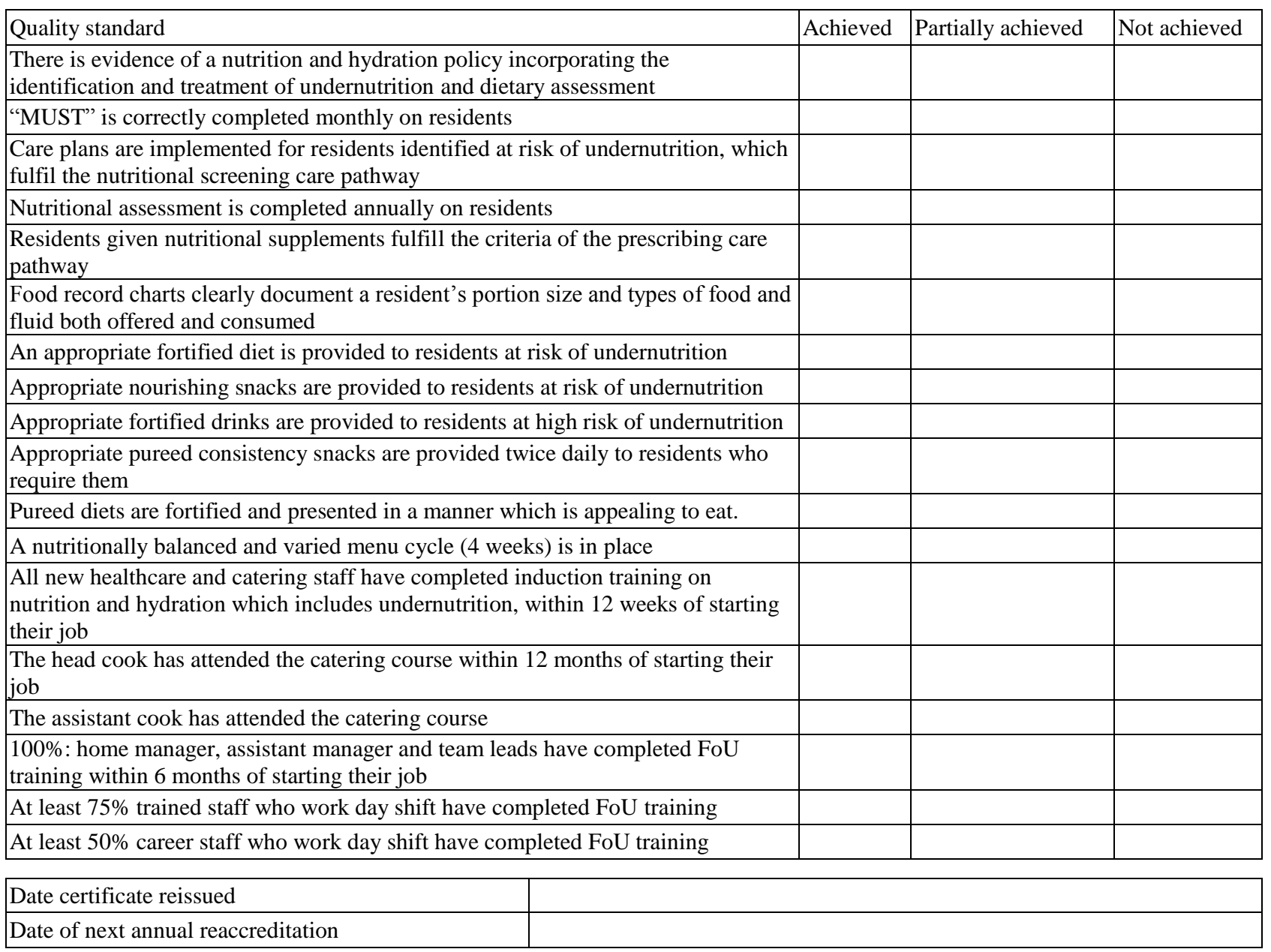


Appendix 2: Overview of the stages of FoU service delivery in care homes

1. Inform the home manager about FoU service

- Information leaflet provided.

(Role: Dietetic Assistant)

2. Written consent from home manager to participate in FoU, includes:

- Permission to collect anonymous baseline, evaluation data from resident records.

- $100 \%$ leaders, $75 \%$ trained staff, $50 \%$ of care staff complete FoU healthcare training.

- Head cook and assistant cook complete FoU catering course.

- The home implements the requirements of the FoU training for identifying and treating undernutrition using FoU "MUST" and FoU care pathways.

(Role: Dietetic Assistant)

\section{Catering course}

- Head cook and assistant cook attend a six week FoU course on menu planning and special diet at a local catering college. (Role: Specialist Dietitian, supported by a Dietetic Assistant)

4. Baseline data collection

- Pseudonymised nutritional care data collected from residents case notes using standards data collection sheet [Appendix 3].

- Quantitative and qualitative data collected on nutrition processes through a structured interview with home manager, cook and observations.

- Audit against undernutrition quality standards （Role: Dietetic Assistant)

5. Health care training

- Health care staff attend an in-house workshop (2 hours) followed by completion of a distance learning workbook (3 hours). Training evaluation forms completed.

- Provision of FoU resources after final workshop: FoU "MUST", FoU guidelines and resource file, fortified recipe book

(Role: Dietetic Assistant)

6. One month review at the care home

Collect completed workbooks

- Monitor progress in implementing 'MUST', nutrition care plans and FoU

(Role: Dietetic Assistant)

\section{Two month review at the care home}

- Return marked workbooks with training certificates

- Monitor progress in implementing 'MUST', nutrition care plans and FoU

(Role: Dietetic Assistant)

8. Six month evaluation

- Collect the same data as outlined at baseline.

- Manager completes service user survey on FoU service

(Role: Dietetic Assistant)

9. Annual review (12 months following end of training)

- Audit and evaluate the home on the undernutrition quality standards. If fail, action plan agreed, which may include provide support and refresher and new staff training.

- When achieved quality standards, issue annual certificate.

- Inform Local Authority commissioners for funding.

(Role: Dietetic Assistant)

10. Annual review (completed annually)

- Continue annual reviews and self assessments every year as outlined.

- Issue annual certificate if fulfil FoU minimum standards and inform Local Authority.

(Role: Dietetic Assistant) 
Appendix 3: Standardised data collection sheet

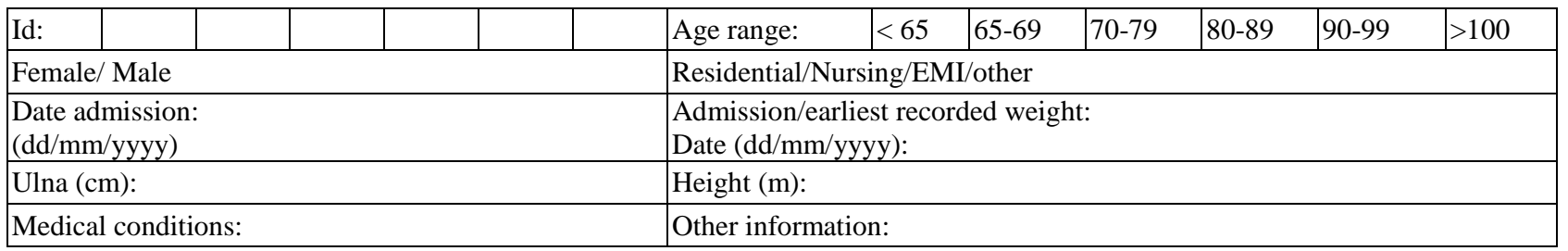

1. CNS (e.g. stroke, dementia, Parkinson's Disease, MS), 2. GI Disease 3. Respiratory Disease, 4. Cardio-vascular disease, 5. Genito/Renal Disease, 6. Musculoskeletal (Including orthopaedic) e.g. post \#NOF, 7. Frail Elderly, 8. Mental Health Disorders (e.g. anxiety, depression, psychosis), 9. Sensory Impairment (e.g. hearing, balance, sight), 10. Cancer. 11. Other.

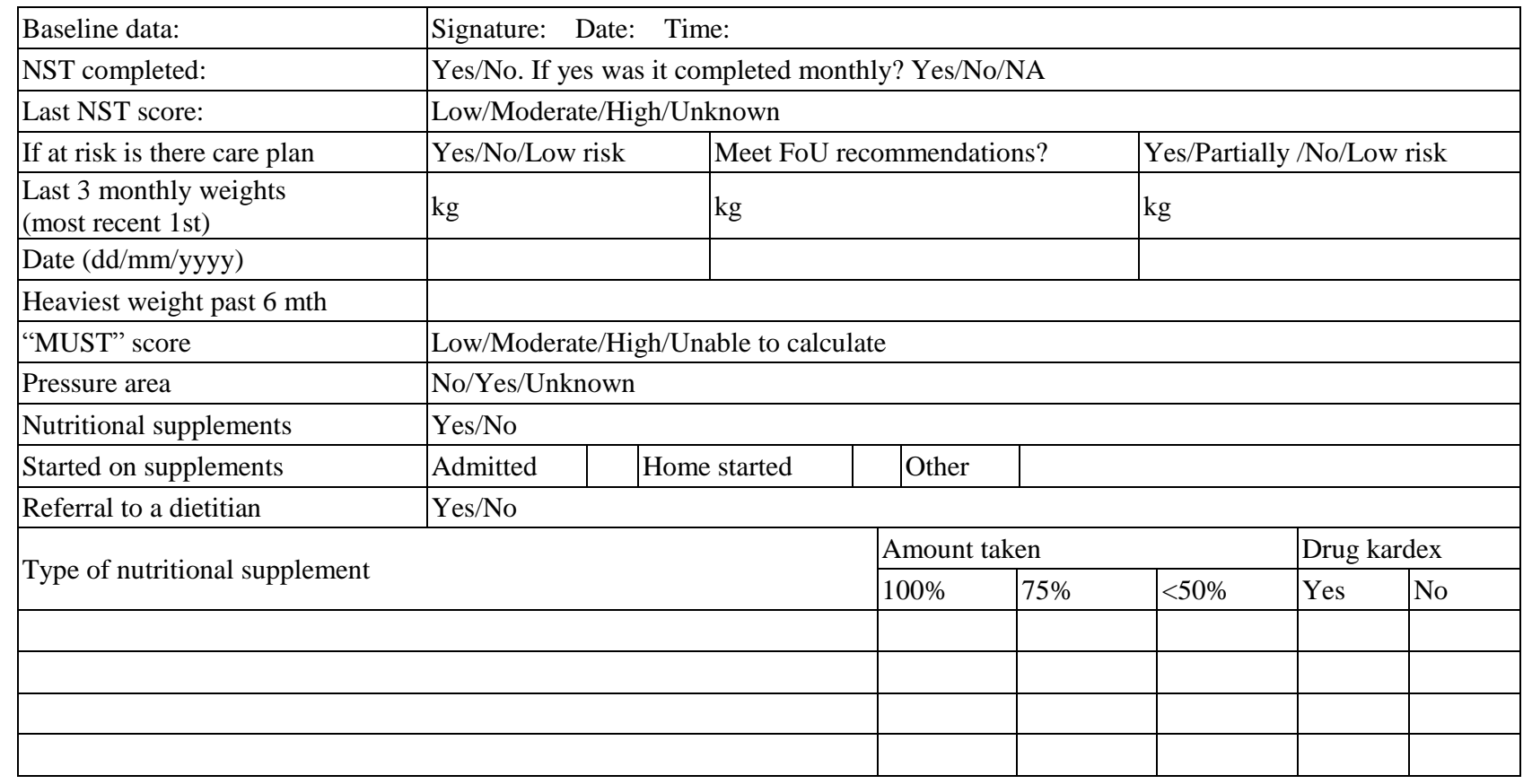

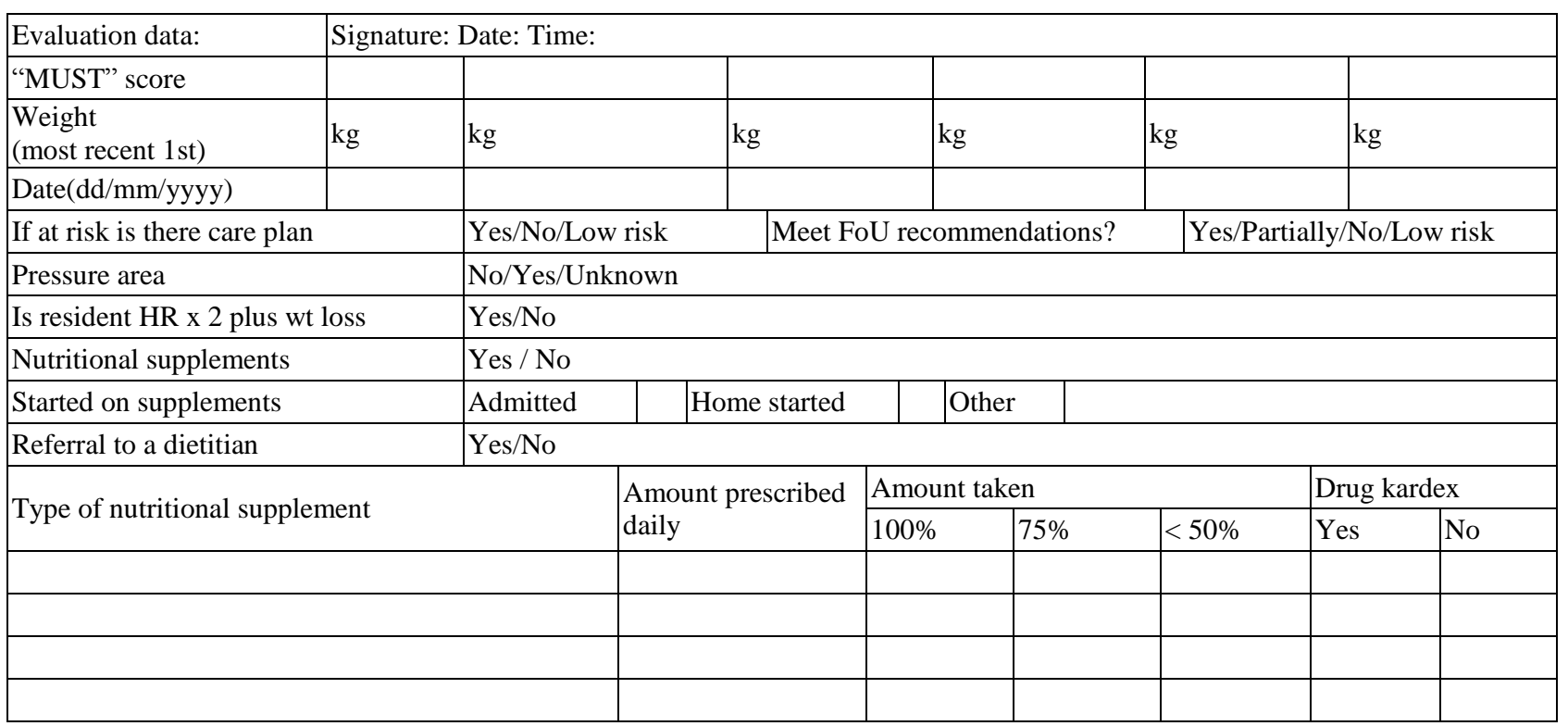

